TRANSACTIONS OF THE

AMERICAN MATHEMATICAL SOCIETY

Volume 358, Number 11, November 2006, Pages 4981-5001

S 0002-9947(06)03883-9

Article electronically published on June 15, 2006

\title{
PARTITIONING $\alpha$-LARGE SETS: SOME LOWER BOUNDS
}

\author{
TERESA BIGORAJSKA AND HENRYK KOTLARSKI
}

\begin{abstract}
Let $\alpha \rightarrow(\beta)_{m}^{r}$ denote the property: if $A$ is an $\alpha$-large set of natural numbers and $[A]^{r}$ is partitioned into $m$ parts, then there exists a $\beta$ large subset of $A$ which is homogeneous for this partition. Here the notion of largeness is in the sense of the so-called Hardy hierarchy. We give a lower bound for $\alpha$ in terms of $\beta, m, r$ for some specific $\beta$.
\end{abstract}

This paper is a continuation of our work [2] and [3] on partitions of finite sets, where the notion of largeness is in the sense of Hardy hierarchy. We give some lower bounds for partitions. All the definitions involving ordinals below $\varepsilon_{0}$, fundamental sequences, the notion of $\alpha$-largeness, etc. are defined in [2]. In order to avoid repetition we assume the reader to have a copy of 2 in hand. We define only the notions needed, which do not occur in 2 .

We stress that the ideas below go back to J. Ketonen and R. Solovay [10. Because of the nature of their problem, that is, describing the order of growth of the function shown by J. Paris and L. Harrington [17 to grow faster than any recursive function provably total in Peano arithmetic, they were interested merely in the existence of $\omega$-large homogeneous sets. We generalize this to higher $\beta$. We use some serious technical simplifications of the ideas of [10] from section 6.3 in [9]. On a more personal level we are highly influenced by the work of Z. Ratajczyk; see [18, [13, [14] and his final [19. In particular, the idea of the notion of arbitrary set of natural numbers (not only interval) being $\alpha$-large is due to Ratajczyk. It should be noted that the idea of Hardy hierarchy was developed by several schools; see, e.g., 7, 8 and [6. The idea of working with $\alpha$-large sets may be used to eliminate the so-called cut elimination from the proofs of consistencies of theories like Peano arithmetic; see Ratajczyk's papers mentioned above and [20, 21] and [1. See also [10, 2] and 3] for some upper bounds for this sort of Ramsey theorem.

The new (when compared with [10] and other papers in this area of combinatorics) idea is to use expansions of ordinals to bases other than $\omega$. The estimation lemma is the basic tool which allows us to estimate how large a homogeneous set is, despite the fact that partitions were constructed with the use of expansions to bases of the form $\omega_{m}$.

We have organized the paper as follows. In section 1 we give the basic construction of partitions of finite sets of natural numbers to be generalized in later sections. The results are well known; we included this section for motivational purposes. In section 2 we give a proof of some version of results due to J. Ketonen and

Received by the editors April 26, 2004 and, in revised form, October 18, 2004.

2000 Mathematics Subject Classification. Primary 05A18.

Key words and phrases. Ramsey theorem, Hardy hierarchy, $\alpha$-large sets.

(C)2006 American Mathematical Society 4981

Reverts to public domain 28 years from publication 
R. Solovay. Our treatment in this section is a rework of section 6.3 in [9, a slightly different treatment is in [16] and 22. In section 3 we prove the estimation lemma which is the main new ingredient in this sort of combinatorics. The referee pointed out that the result of this section is closely related to the result of [4]. In the final section, section 4, we give our main results. Thus, we mimic the arguments from sections 1 and 2 and give a construction of our partitions, and use the estimation lemma to show that these partitions do not admit too large homogeneous sets.

The notion of a set being $\alpha$-large is defined in 2. We shall say that a set is $\alpha$-small if it is not $\alpha$-large. We say that a set $A \subset \mathbb{N}$ is exactly $\alpha$-large if it is $\alpha$-large, but $A \backslash\{\max (A)\}$ is $\alpha$-small. Finally, say that a set is at most $\alpha$-large if it is exactly $\alpha$-large or $\alpha$-small. Some more notation concerning ordinals, largeness and everything connected with it occurs in the body of the paper.

The authors would like to thank Dr. B. Piekart for pointing out several inaccuracies in earlier drafts of this paper and to Mr. Konrad Zdanowski for discussions on the topics contained in our work. Finally we would like to thank the referee for giving us more bibliographical information.

\section{Some lower Bounds: Classic}

The purpose of this section is motivational. We give some lower bounds for the finite Ramsey theorem. The bounds we give are very weak, but in section 4 we shall modify the proofs given below. For far stronger results concerning the finite Ramsey theorem, see [9] in particular section 4.2 in 9] for a nonconstructive method of proving the existence of partitions of pairs admitting no large homogeneous sets (by the way, one needs the so-called Stirling formula to make the proof in 9] complete) and section 4.7 in [9] for the stepping up lemma which allows one to obtain lower bounds for partitioning $k$-tuples, $k>2$.

The starting point of our considerations is the following simple construction. Let $A$ be any set of cardinality at most $m^{n}$. We may identify $A$ with a subset of $\{0, \cdots, m-1\}\{0, \cdots, n-1\}$. One may work with expansions (to the base $m$ ) of numbers smaller than $m^{n}$ or with set of sequences of length $n$ of numbers smaller than $m$. We shall take this second possibility. Thus, we regard $A$ as a subset of the family of sequences as above. For $a, b \in A$ we put

$$
P(a, b)=\min j: a_{j} \neq b_{j}
$$

and treat $P$ as a partition of $[A]^{2}$ into $n$ parts.

Lemma 1.1. If $D \subseteq A$ is homogeneous for $P$, then $\operatorname{Card}(D) \leq m$.

Proof. Let $D$ be monochromatic under $P$. Hence there exists $j<n$ such that $\forall(a, b) \in[D]^{2} a_{j} \neq b_{j}$. It follows that $D$ has at most $m$ elements, indeed, there are at most $m$ possible $d_{j}$ for $d \in D$.

If we partition triples, then the number of parts may be reduced to 3 for a little price. We let

$$
K(a, b, c)=\left\{\begin{array}{lll}
0 & \text { if } & P(a, b)>P(b, c) \\
1 & \text { if } & P(a, b)=P(b, c) \\
2 & \text { if } & P(a, b)<P(b, c)
\end{array}\right.
$$

We shall write $P^{A}$ and $K^{A}$ if needed. 
Lemma 1.2. Under the notation and assumption as above, if $D$ is homogeneous for $K$, then $\operatorname{Card}(D) \leq \max (m, n+1)$. To be more specific, if $[D]^{3}$ gets color 1 , then $\operatorname{Card}(D) \leq m$, and if $[D]^{3}$ gets color 0 or 2 , then $\operatorname{Card}(D) \leq n+1$.

Proof. We let $D=\left\{d_{0}, \cdots, d_{r-1}\right\}$. If the color of $[D]^{3}$ is 0 or 2 , then we put $j_{i}=P\left(d_{i}, d_{i+1}\right)$. It follows that the sequence $j_{i}: i<r-2$ is strictly monotonic, hence one-to-one, and hence it has at most $n$ elements, so $D$ has at most $n+1$ elements. If this color is 1 , then $j=P\left(d_{i}, d_{i+1}\right)$ does not depend on $i$. Moreover, the sequences $d_{i}, d_{i+1}$ are equal at coordinates corresponding to indices $0, \cdots, j-1$, but their $j$-th coordinates are different. These coordinates form a strictly increasing sequence by the properties of the lexicographic ordering of the product. Thus, there are at most $m$ candidates for the $j$-th coordinate of $d_{i}$, so there are at most $m$ possible $d_{i}$ 's.

Before giving the general inductive step of the construction of partitions that we have in mind, we give the step $k=4$ for getting a better idea of what will happen. Thus we shall construct a partition of $[A]^{4}$. The assumption on $A$ is that its cardinality is at most $m^{n^{n}}$. Thus, we identify $A$ with the set of sequences of length $n^{n}$ of numbers smaller than $m$.

For $a=\left(a^{0}, a^{1}, a^{2}, a^{3}\right) \in[A]^{4}$ we let $G(a)=K^{A}\left(a^{0}, a^{1}, a^{2}\right)$. We defined a partition $G$ of $[A]^{4}$ into 3 parts. Observe that if $D \subseteq A$ is of cardinality strictly greater than 4 and is homogeneous for it, then $K^{A}$ is constant on $[D \backslash\{\max (D)\}]^{3}$.

We define an additional partition $R$ of $[A]^{4}$ in the following way. For $a \in[A]^{4}$ we let $b_{i}=P\left(a^{i}, a^{i+1}\right)$. Let $B$ be the natural set of cardinality $n^{n}$, i.e., the set of all sequences of length $n$ of numbers $0, \cdots, n-1$. Then each $b^{i}$, being smaller than the length of sequences from $A$, is an element of $B$. Let $a \in[A]^{4}$. We let

$$
R(a)=\left\{\begin{array}{lll}
K^{B}\left(b_{0}, b_{1}, b_{2}\right) & \text { if } & b_{0}<b_{1}<b_{2}, \\
K^{B}\left(b_{2}, b_{1}, b_{0}\right) & \text { if } & b_{0}>b_{1}>b_{2}, \\
0 & & \text { in other cases }
\end{array}\right.
$$

where $b_{i}=P\left(a^{i}, a^{i+1}\right)$ as above. Finally we put

$$
L(a)=\langle G(a), R(a)\rangle .
$$

Obviously, $L$ is a partition of $[A]^{4}$ into $3^{2}$ parts.

Lemma 1.3. Under the above assumption (i.e., $\operatorname{Card}(A) \leq m^{n^{n}}$ ) we have: whenever $D \subseteq A$ is homogeneous for $L$, then $\operatorname{Card}(D) \leq \max (m+1, n+3)$.

Proof. Let $D \subseteq A$ be homogenous for $L$. Then $D$ is homogeneous for both $G, R$. Write $D=\left\{d_{0}, \cdots, d_{r-1}\right\}$ in increasing order. Then $D^{\prime}=D \backslash\{\max (D)\}=$ $\left\{d_{0}, \cdots, d_{r-2}\right\}$ is homogeneous for $K$. If $K^{A}$ colors $D^{\prime}$ by 1 , then $\operatorname{Card}(D) \leq m+1$ by Lemma 1.2. If $D$ gets color 0 or 2 , then the sequence $b^{i}=P\left(d^{i}, d^{i+1}\right)$ is either strictly increasing or strictly decreasing. In both cases, $K^{B}$ is constant on the set of $b_{i}$ 's. By Lemma 1.2, $B$ has at most $n+1$ elements, hence $D^{\prime}$ has at most $n+2$ elements, so $D$ has at most $n+3$ elements.

Let us describe further steps of construction of partitions. As we shall need the function which associates $n^{.{ }^{n}}$ with $n$ (the tower of $k n$ 's), let us introduce a notation for it. Let $\operatorname{tow}_{0}(n)=1$ and $\operatorname{tow}_{k+1}(n)=n^{\text {tow }_{k}(n)}$. 
Theorem 1.4. For every $k>2$, every $m, n$ and every set $A$ of cardinality at most $m^{\text {tow }_{k-2}(n)}$, there exists a partition $L$ of $[A]^{k}$ into $3^{k}$ parts such that every homogeneous set is of cardinality at most $\max \left(m+k-3, n+\frac{1}{2}(k-1) \cdot(k-2)\right)$.

Proof. We proceed by induction on $k \geq 3$; for $k=3$ the result was proved above. Assume the result for $k$. Let $A$ have at most $m^{\text {tow }_{k-1}(n)}$ elements. We regard it as the set of sequences of length tow $k-1(n)$ of numbers up to $m-1$. Let the partition $P^{A}$ be given by (11) and let $K^{A}$ be defined by (2). Let $B$ be the set of values of $P$. We define the partition $G$ as before, i.e., $G(a)=K^{A}\left(a_{0}, a_{1}, a_{2}\right)$ for $a \in[A]^{k+1}$. Once again, if a subset $B$ of $A$ is homogeneous for $G$, then $B$ without its last $k-2$ elements is monochromatic with respect to $K^{A}$. Let $W$ be the partition of $[B]^{k}$ given by the inductive assumption (with $m=n$ ). We let $b_{i}=P\left(a_{i}, a_{i+1}\right)$. We define the partition $L$ exactly as above, but now we work with $k$-tuples rather than triples. That is, we put

$$
R(a)=\left\{\begin{array}{lll}
W\left(b_{0}, \cdots, b_{k-1}\right) & \text { if } & b_{0}<\cdots<b_{k-1} \\
W\left(b_{k-1}, \cdots, b_{0}\right) & \text { if } & b_{0}>\cdots>b_{k-1} \\
0 & & \text { in other cases. }
\end{array}\right.
$$

We ask the reader to check that the partition $L$ given by

$$
L(a)=\langle G(a), R(a)\rangle
$$

has the desired property. We merely point out that if $D$ is homogeneous for $L$, then $D^{\prime}=D$ without its last $k-2$ elements is homogeneous for $G$. It follows that if the value of $G$ on $[A]^{k+1}$ is 1 , then $\operatorname{Card}\left(D^{\prime}\right) \leq m$, and if the appropriate value is 0 or 2 , then the set $B=\left\{P\left(d_{i}, d_{i+1}\right): i\right\}$ has the cardinality estimated by the cardinality of sets homogeneous for $W$.

\section{The Ketonen-Solovay Case}

In this section we give a variant of the results due to Ketonen and Solovay. Our results are weaker than those of [10, but in section 4 we shall generalize the construction presented below. As pointed out in the introduction, the material of this section is a rework of section 6.3 in [9]; cf. also [16]. See also [22.

Let $\alpha<\varepsilon_{0}$. Then $\alpha$ may be written in the Cantor normal form

$$
\alpha=\omega^{\alpha_{0}} \cdot a_{0}+\cdots+\omega^{\alpha_{s}} \cdot a_{s}
$$

for some $\alpha_{0}>\alpha_{1}>\cdots>\alpha_{s}$ with $\alpha>\alpha_{0}$ and $a_{0}, \cdots, a_{s} \in \mathbb{N} \backslash\{0\}$.

$\operatorname{By} \operatorname{LM}(\alpha)$ we denote the leftmost exponent of $\alpha$, that is, if $\alpha$ is written in the form (6), then $\operatorname{LM}(\alpha)=\alpha_{0}$. Similarly, by $\operatorname{RM}(\alpha)$ we denote the rightmost exponent of $\alpha$, i.e. $\alpha_{s}$ is (6). (In [2] we denoted $\operatorname{RM}(\alpha)$ by $\varrho(\alpha)$.)

As in the literature we write $\beta \gg \alpha$ if either $\alpha=0$ or $\beta=0$, or all the exponents in the Cantor normal form of $\beta$ are $\geq$ all the exponents in the normal form of $\alpha$. We write $\beta \ggg \alpha$ for the same but with strict inequality.

We shall also use the so-called short normal form of $\alpha$. It is defined as follows. By putting $\beta=\omega^{\alpha_{0}} \cdot a_{0}+\cdots+\omega^{\alpha_{s}} \cdot\left(a_{s}-1\right)$ and $\alpha_{s}=\xi$ we see that $\alpha$ may be written as

$$
\alpha=\beta+\omega^{\xi},
$$

where $\beta \gg \omega^{\xi}$. We shall refer to (7) as to the short Cantor normal form of $\alpha$.

Observe that if $\alpha$ is written in its Cantor normal form (6), then all exponents may be written in their Cantor normal forms, etc. This process (when iterated 
sufficiently many times so that only natural numbers occur in the expansion) yields the full Cantor normal form of $\alpha$.

We define the pseudonorm of $\alpha$ as the greatest natural number which occurs in its (full) Cantor normal form. Technically we define the function psn sending ordinals below $\varepsilon_{0}$ into $\mathbb{N}$ by putting $\operatorname{psn}(n)=n$ for $n<\omega$ and for $\alpha \geq \omega$

$$
\operatorname{psn}(\alpha)=\max \left(\operatorname{psn}\left(\alpha_{0}\right), \cdots, \operatorname{psn}\left(\alpha_{s}\right), a_{0}, \cdots, a_{s}\right),
$$

where $\alpha$ is written in its Cantor normal form (6).

The following properties of pseudonorm are easy:

1. If $\mu \gg \nu$, then $\operatorname{psn}(\mu+\nu) \geq \operatorname{psn}(\mu), \operatorname{psn}(\nu)$,

2. $\operatorname{psn}\left(\omega^{\rho}\right)=\operatorname{psn}(\rho)$.

We ask the reader to check these properties. We merely point out that the inequality in point 1 may be strict (and if this happens, then $\operatorname{RM}(\mu)=\operatorname{LM}(\nu)$ ). We write "pseudonorm" because Ketonen and Solovay [10] use a slightly different function, the norm of $\alpha$ for a similar purpose. The property is as follows.

Lemma 2.1. For every limit $\beta<\varepsilon_{0}$ we have:

$$
\forall \alpha<\beta \forall m>1\left\{\operatorname{psn}(\alpha)<m \Rightarrow\left[\alpha<\{\beta\}(m) \&\{\beta\}(m) \Rightarrow_{m} \alpha\right]\right\} .
$$

We remark that the weaker assumption $\operatorname{psn}(\alpha) \leq m$ is too weak. For example, let $\beta=\omega^{2}, \alpha=\omega \cdot 5+5$ and $m=5$.

Proof of Lemma 2.1. We proceed by induction on $\beta$. If $\beta=\omega$, then $\alpha$ is a natural number which is smaller than $\{\beta\}(m)$ because its pseudonorm is smaller. For $\beta>\omega$ we proceed by induction on $\operatorname{RM}(\beta)$. That is, we let $T(\beta)$ denote the statement in the lemma and prove by induction on $\xi$ that $\forall \xi W(\xi)$, where $W(\xi)$ is

$$
\forall \beta\left\{\left[\operatorname{RM}(\beta) \leq \xi \& \forall \beta^{\prime}<\beta T\left(\beta^{\prime}\right)\right] \Rightarrow T(\beta)\right\} .
$$

Let $\xi=1$. Let $\beta$ be such that $\operatorname{RM}(\beta)=1$. Then $\beta$ is of the form $\varrho+\omega$ for some $\varrho \gg \omega$. Let $\alpha<\beta$ and $\operatorname{psn}(\alpha)<m$.

CASE 1. $\alpha<\varrho$. Then obviously $\alpha<\{\beta\}(m)=\varrho+\{\omega\}(m)=\varrho+m$. For the second claim we apply the inductive assumption $T(\varrho)$ and obtain $\varrho \Rightarrow_{m} \alpha$. Moreover, we also have $\varrho+m \Rightarrow_{m} \varrho$. Concatenation of the resulting sequences witnesses $\beta \Rightarrow_{m} \alpha$.

CASE 2. $\varrho \leq \alpha$. Then $\alpha$ must be of the form $\varrho+\mu$ for some $\mu$. Also, $\mu<\omega$ and $\operatorname{psn}(\mu)<m$. It follows that $\alpha=\varrho+k$ for some $k<m$. But then both conclusions are immediate.

Assume $W(\xi)$; we want to prove $W(\xi+1)$. So let $\operatorname{RM}(\beta)=\xi+1$. Then $\beta=\varrho+\omega^{\xi+1}$ for some $\varrho \gg \omega^{\xi+1}$. Pick $\alpha, m$ satisfying the assumption of $W(\xi+1)$. Observe that $\{\beta\}(m)=\varrho+\omega^{\xi} \cdot m$.

CASE $1 . \alpha<\varrho$. We apply the inductive assumption $T(\varrho)$ as above.

CASE 2. $\varrho \leq \alpha$. (In particular, if $\varrho=0$, then this case holds.) Then $\alpha$ must be of the form $\alpha=\varrho+\tau$ for some $\tau<\omega^{\xi+1}$. Moreover $\operatorname{psn}(\tau)<m$. It suffices to show that

$$
\tau<\left\{\omega^{\xi+1}\right\}(m)=\omega^{\xi} \cdot m \text { and } \omega^{\xi} \cdot m \Rightarrow_{m} \tau .
$$

If $\varrho \neq 0$, then this follows immediately from $T\left(\omega^{\xi+1}\right)$. So let $\varrho=0$, then we derive this fact directly. We write $\tau=\omega^{\xi} \cdot t+\mu$, where $\mu \lll \omega^{\xi}$ ( $t$ may be equal to 0 ). Obviously, $t<m$, because $t \leq \operatorname{psn}(\tau)<m$, hence $\tau<\omega^{\xi} \cdot m$, and hence it suffices to show the second claim. We write $\omega^{\xi} \cdot m=\omega^{\xi} \cdot t+\omega^{\xi} \cdot(m-t)$. Now we have $\omega^{\xi} \cdot(m-t) \Rightarrow_{m} \omega^{\xi}$ and $\omega^{\xi} \Rightarrow_{m} \mu$ by $T\left(\omega^{\xi}\right)$. 
Let $\xi$ be the limit and assume $\forall \xi^{\prime}<\xi W\left(\xi^{\prime}\right)$. Let $\beta$ be such that $\operatorname{RM}(\beta)=\xi$. Once again, $\beta=\varrho+\omega^{\xi}$ for some $\varrho \gg \omega^{\xi}$. Let $\alpha<\varrho+\omega^{\xi}$ and $m>\operatorname{psn}(\alpha)$.

CASE $1 . \alpha<\varrho$. Then by $T(\varrho)$ we have $\alpha<\varrho$ and $\{\varrho\}(m) \Rightarrow_{m} \alpha$, and both claims follow by the same reasoning as above.

CASE 2. $\varrho \leq \alpha$. Then $\alpha=\varrho+\tau$ for some $\tau \ll \varrho$. Once again, if $\varrho \neq 0$, then we apply $T\left(\omega^{\xi}\right)$, so assume that $\varrho=0$. Thus we must show that $\tau<\omega^{\{\xi\}(m)}$ and $\omega^{\{\xi\}(m)} \Rightarrow_{m} \tau$ from the assumptions that $\tau<\omega^{\xi}$ and $m>\operatorname{psn}(\tau)$. We write $\tau=\omega^{\tau_{0}} \cdot t_{0}+\cdots+\omega^{\tau_{r}} \cdot t_{r}$ in the Cantor normal form. We must have $\tau_{0}<\xi$. We apply $T(\xi)$ to infer $\xi \Rightarrow_{m} \tau_{0}$, and, hence, $\omega^{\xi} \Rightarrow_{m} \omega^{\tau_{0}}$ by the results of Ketonen and Solovay [10] as collected in 2] (lemma 2, part (iv) in [2]). In particular, $\{\xi\}(m) \geq \tau_{0}$. We assert that this inequality is strict. It suffices to show that $\operatorname{psn}(\{\xi\}(m)) \geq m$. We verify this observation by induction on $\xi$. This fact obviously holds for $\xi=\omega$, and, indeed, for $\xi$ of the form $\omega^{\alpha+1}$. Further, for the limit $\lambda,\left\{\omega^{\lambda}\right\}(m)=\omega^{\{\lambda\}(m)}$, so the inequality holds for $\omega^{\lambda}$, provided it holds for $\lambda$. Finally, if $\xi$ has nontrivial Cantor normal form expansion, the result is immediate because fundamental sequences are defined using the rightmost item of this expansion. Thus, $\{\xi\}(m)>\tau_{0}$, and hence $\tau<\left\{\omega^{\xi}\right\}(m)$. The second part follows from the inductive assumption $T\left(\omega^{\{\xi\}(m)}\right)$.

Lemma 2.1 has the following corollary.

Corollary 2.2. If $\beta$ is limit, $\alpha<\beta$ and $A \subset \mathbb{N}$ is $\beta$-large and satisfies $\min (A)>$ $\operatorname{psn}(\alpha)>1$, then $A$ is $\alpha$-large.

Proof. It suffices to show that under the assumption of the corollary, $h_{\alpha}\left(a_{0}\right) \downarrow$, where $a_{0}=\min (A)$ as usual. We apply the second part of the conclusion of Lemma 2.1 to $m=a_{0}$ and the results due to Ketonen-Solovay [10; see lemma 4, part (ii) in [2].

Our first goal is to construct a sequence of partitions of ordinals below $\varepsilon_{0}$. It will be convenient to have some more notation concerning ordinals. We let $v_{\delta}(\alpha)$ be the coefficient of $\omega^{\delta}$ in the Cantor normal expansion of $\alpha$ (and $v_{\delta}(\alpha)=0$ if $\omega^{\delta}$ does not occur in this expansion). Thus, if $\alpha$ is written in the form (6), then $v_{\alpha_{i}}(\alpha)=a_{i}$ for all $i \leq s$ and $v_{\delta}(\alpha)=0$ for other $\delta$. In particular, $v_{\delta}(0)=0$ for every $\delta$.

For ordinals $\alpha, \beta<\varepsilon_{0}$ we also define the leftmost difference in the expansions of ordinals $\alpha, \beta$ by

$$
\operatorname{LD}(\alpha, \beta)=\max \delta: v_{\delta}(\alpha) \neq v_{\delta}(\beta) .
$$

We treat LD as a partition of $\left[\varepsilon_{0}\right]^{2}$ whose values are ordinals. Let us make a convention that when working with finite sets of ordinals we write them in decreasing order. This will be convenient. Indeed, later we shall transfer partitions of sets of ordinals to partitions of sets of natural numbers by means of an order-reversing function, so this will fit the general scheme of writing sets of natural numbers in increasing order.

Lemma 2.3. Let $D$ be a set of ordinals homogeneous for LD. Then $D$ has at most $\operatorname{psn}(\max (D))+1$ elements.

Proof. Let $D$ be monochromatic for LD. Thus, there exists $\nu<\varepsilon_{0}$ such that $\forall(\alpha, \beta) \in[D]^{2} \operatorname{LD}(\alpha, \beta)=\nu$. Let $D=\left\{\delta_{0}, \cdots, \delta_{r-1}\right\}$ be a decreasing enumeration of $D$. Let $\delta_{i}=\varrho_{i}+\omega^{\nu} \cdot m_{i}+\tau_{i}$ in the Cantor normal form. Then $\varrho_{i}$ does not depend on $i$, indeed, if $\varrho_{i} \neq \varrho_{j}$, then $\operatorname{LD}\left(\delta_{i}, \delta_{j}\right)>\nu$. Moreover, the sequence $m_{i}$ must be 
one-to-one, for if $m_{i}=m_{j}$, where $i \neq j$, then $\operatorname{LD}\left(\delta_{i}, \delta_{j}\right)<\nu$. The sequence $m_{j}$ must be strictly decreasing. Thus, the cardinality of $D$ cannot exceed $m_{0}+1$. Also, clearly, $m_{0} \leq \operatorname{psn}\left(\delta_{0}\right)$.

The second partition of ordinals needed in the sequel is the first one of the promised sequence. It is defined as follows:

$$
\mathrm{KE}(\alpha, \beta, \gamma)=\left\{\begin{array}{lll}
0 & \text { if } & \mathrm{LD}(\alpha, \beta)<\operatorname{LD}(\beta, \gamma), \\
1 & \text { if } & \operatorname{LD}(\alpha, \beta)=\operatorname{LD}(\beta, \gamma), \\
2 & \text { if } & \operatorname{LD}(\alpha, \beta)>\operatorname{LD}(\beta, \gamma)
\end{array}\right.
$$

This is a partition of $\left[\varepsilon_{0}\right]^{3}$ into 3 parts. The basic property of KE is that if $D \subseteq \omega^{\omega}$ is homogeneous, then $\operatorname{Card}(D) \leq \operatorname{psn}(\max (D))+2$. But we shall need a more detailed description.

Lemma 2.4. (i) If $D$ is such that $\mathrm{KE}$ colors $[D]^{3}$ by 1 , then $D$ has at most $\operatorname{psn}(\max (D))+1$ elements. (ii) If $D$ is such that $\max (D)<\omega^{\omega}$ and $[D]^{3}$ is colored by 0 or 2 , then $D$ has at most $\operatorname{psn}(\max (D))+2$ elements.

Proof. The first part is checked almost exactly like Lemma 2.3. Once again, we write $D=\left\{\delta_{0}, \cdots, \delta_{r-1}\right\}$ in decreasing order and $\delta_{i}=\varrho_{i}+\omega^{\nu} \cdot m_{i}+\tau_{i}$, where $\nu$ is such that $\operatorname{LD}\left(\delta_{i}, \delta_{i+1}\right)=\nu$ for all $i$. Then $\varrho_{0}=\varrho_{1}$ because $\operatorname{LD}\left(\delta_{0}, \delta_{1}\right)=\nu$, etc. Also the same reasoning as in the proof of Lemma 2.3 shows that the sequence $m_{i}$ is strictly decreasing, and the first part follows.

For the second part let $D$ be enumerated as above; assume moreover that $\delta_{0}<$ $\omega^{\omega}$. Let $\gamma_{i}=\operatorname{LD}\left(\delta_{i}, \delta_{i+1}\right)$ for $i<r-1$. The sequence $\gamma_{i}$ consists of natural numbers (because they are exponents of ordinals smaller than $\omega^{\omega}$ ), and by the assumption of the case this sequence is either strictly increasing or strictly decreasing. If it is strictly decreasing, then there at most $\operatorname{psn}\left(\delta_{0}\right)+1$ of $\gamma_{i}$ 's, so $\operatorname{Card}(D) \leq \operatorname{psn}\left(\delta_{0}\right)+2$. Assume that the sequence $\gamma_{i}: i<r-1$ is increasing. We write $\delta_{0}=\omega^{u} \cdot w+\varrho_{0}+$ $\omega^{\gamma_{0}} \cdot m_{0}+\tau_{0}$ with $\omega^{u} \ggg \varrho_{0} \ggg \omega^{\gamma_{0}} \ggg \tau_{0}$ and consider the sequence $u-\gamma_{i}$. It is strictly decreasing and has items less than or equal to $u$, so less than or equal to $\operatorname{psn}\left(\delta_{0}\right)$. It follows that $r-1 \leq \operatorname{psn}\left(\delta_{0}\right)+1$, i.e. $r \leq \operatorname{psn}\left(\delta_{0}\right)+2$ as desired.

Let $\omega_{1}=\omega, \omega_{m+1}=\omega^{\omega_{m}}$.

Lemma 2.5. Let $k \geq 3$. Then there exists a partition $L_{k}$ of $\left[\varepsilon_{0}\right]^{k}$ into $3^{k-2}$ parts such that whenever $D \subset \omega_{k-1}$ is homogeneous for $L_{k}$, then

$$
\operatorname{Card}(D) \leq \operatorname{psn}(\max (D))+\frac{(k-1) \cdot(k-2)}{2}+1 .
$$

Proof. The partition $L_{3}=\mathrm{KE}$ satisfies our demand for $k=3$ as verified above. Suppose we are given a partition $L_{k}$ of $\left[\varepsilon_{0}\right]^{k}$ into $3^{k-2}$ parts; we construct a partition $L_{k+1}$. For $\alpha=\left\langle\alpha^{0}, \cdots, \alpha^{k}\right\rangle \in\left[\varepsilon_{0}\right]^{k+1}$ we let $G(\alpha)=L_{3}\left(\alpha^{0}, \alpha^{1}, \alpha^{2}\right)$. Thus $G$ does not depend on $\alpha^{3}, \cdots, \alpha^{k}$.

Assume that $D \subseteq \varepsilon_{0}$ is homogeneous for $G$. Let $D^{\prime}$ denote $D$ without its last (i.e. smallest) $k-2$ elements. Let us denote by $\delta_{3}, \cdots, \delta_{k}$ these last elements of $D$. We assert that $D^{\prime}$ is homogeneous for $L_{3}$. Indeed, for $\left(\xi^{0}, \xi^{1}, \xi^{2}\right) \in\left[D^{\prime}\right]^{3}$ we have $L_{3}\left(\xi^{0}, \xi^{1}, \xi^{2}\right)=G\left(\xi^{0}, \xi^{1}, \xi^{2}, \delta_{3}, \cdots, \delta_{k}\right)$.

We construct an additional partition $W$ of $\left[\varepsilon_{0}\right]^{k+1}$ in the following manner. Given $\alpha=\left\langle\alpha^{0}, \cdots, \alpha^{k}\right\rangle \in\left[\varepsilon_{0}\right]^{k+1}$ we let $\beta(\alpha)$ be the sequence $\beta^{i}=\operatorname{LD}\left(\alpha^{i}, \alpha^{i+1}\right)$. Thus, 
$\beta(\alpha)$ is a sequence of length $k$ which need not be monotonic. We let

$$
W(\alpha)=\left\{\begin{array}{lrr}
L_{k}\left(\beta^{0}, \cdots, \beta^{k-1}\right) & \text { if } & \beta^{0}>\cdots>\beta^{k-1}, \\
L_{k}\left(\beta^{k-1}, \cdots, \beta^{0}\right) & \text { if } & \beta^{0}<\cdots<\beta^{k-1}, \\
0 & \text { otherwise. }
\end{array}\right.
$$

Of course, $\beta$ is the sequence $\beta(\alpha)$ as defined above, that is, $\beta^{i}=\operatorname{LD}\left(\alpha^{i}, \alpha^{i+1}\right)$. We let

$$
L_{k+1}(\alpha)=\langle G(\alpha), W(\alpha)\rangle \text {. }
$$

Obviously, $L_{k+1}$ is a partition of $\left[\varepsilon_{0}\right]^{k+1}$ into $3^{k-1}$ parts. Let $D$ be homogeneous for $L_{k+1}$. Then $D^{\prime}=D$ without $k-2$ last elements of $D$ is homogeneous for $L_{3}$ as observed above. It follows that if $L_{3}$ colors $\left[D^{\prime}\right]^{3}$ by 1 , then $D^{\prime}$ has at $\operatorname{most} \operatorname{psn}(\max (D))+1$ elements, so $D$ has at most $\operatorname{psn}(\max (D))+k-1$ elements, and the conclusion holds. So assume that $L_{3}$ colors $\left[D^{\prime}\right]^{3}$ by 0 or 2 . Then we use the assumption that $D \subset \omega_{k}$ and the homogeneity of $D^{\prime}$ with respect to $W$. To be more specific, we write $D^{\prime}=\left\{\xi_{0}, \cdots, \xi_{r-1}\right\}$ in decreasing order and put $\mu_{i}=\operatorname{LD}\left(\xi_{i}, \xi_{i+1}\right)$ for $i<r-1$. This sequence is either strictly increasing or strictly decreasing by the assumption of the case. The partition $W$ ensures that the set $M=\left\{\mu_{0}, \cdots, \mu_{r-2}\right\}$ is homogeneous for $L_{k}$. Moreover, its elements, being exponents in ordinals below $\omega_{k}$, are ordinals below $\omega_{k-1}$. Hence, by the inductive assumption, $M$ has at most $\operatorname{psn}(\max (M))+\frac{(k-2)(k-1)}{2}+1$ elements, so $D^{\prime}$ has at $\operatorname{most} \operatorname{psn}(\max (M))+\frac{(k-2)(k-1)}{2}+2$ elements. Also, obviously $\operatorname{psn}(\max (M)) \leq$ $\operatorname{psn}(\max (D))$. Hence, the whole $D$ has at most $\operatorname{psn}(\max (D))+\frac{(k-2)(k-1)}{2}+k$ elements, but this number is just $\operatorname{psn}(\max (D))+\frac{k \cdot(k-1)}{2}+1$.

Here is the main consequence of this construction. It is a version of results due to Ketonen and Solovay [10.

Theorem 2.6. Let $k \geq 3$. Let $A \subset \mathbb{N}$ be such that for every partition $L$ : $[A]^{k} \rightarrow 3^{k-2}$ there exists a monochromatic $D \subseteq A$ with strictly more than $\min (D)+$ $\frac{(k-2) \cdot(k-1)}{2}+1$ elements. Then $A$ is $\omega_{k-1}$-large.

In order to prove this result we need some more apparatus. In the definition of a set $A \subset \mathbb{N}$ being $\alpha$-large we used Hardy iterations $h_{\alpha}^{A}$ of $h=h^{A}$, the successor in the sense of $A$; cf. [2, pp. 27 and 28. It may happen that $h_{\alpha}(b)=h_{\beta}(b)$ also if $\alpha \neq \beta$, e.g., $h_{\omega}(b)=h_{b}(b)$. It will be convenient to be able to choose some ordinal uniquely in such situations. This is done as follows. Let a set $A$ be given. Let $\mu<\varepsilon_{0}$. We define two sequences $\mu_{j}, b_{j}$ by the following induction. We let $\mu_{0}=\mu$ and $b_{0}=a_{0}=\min (A)$. Assume that $\mu_{j}$ and $b_{j}$ are constructed. If $\mu_{j}=0$, then the construction terminates. If $\mu_{j}>0$ and $\mu_{j}$ is the limit we let $\mu_{j+1}=\left\{\mu_{j}\right\}\left(b_{j}\right)$ and $b_{j+1}=b_{j}$. If $\mu_{j}$ is the nonlimit, then the construction terminates if $b_{j}=a_{r-1}=$ $\max (A)$, otherwise we let $\mu_{j+1}=\mu_{j}-1$ and $b_{j+1}=h^{A}\left(b_{j}\right)$, the next element of $A$. This completes the definition of the sequences $\mu_{j}, b_{j}$.

Observe that the sequence $\mu_{j}$ is decreasing. We also remark that the construction terminates in two cases: $\mu_{j}=0$ or $\mu_{j}$ is the nonlimit and $b_{j}=\max (A)$. It should also be noted that in the process of this construction we passed from $b_{j}$ to the next element of $A$ only if $\mu_{j}$ is the nonlimit.

The following proposition is (essentially) the original definition of a $\mu$-large set; cf. Ketonen-Solovay [10]. 
Proposition 2.7. Under the notation introduced above, $A$ is $\mu$-large iff there exists $j$ such that $\mu_{j}=0$.

Proof. We claim that the following are equivalent:

1. $A$ is $\mu$-large,

2. for every $j$ the set $\left\{x \in A: b_{j} \leq x\right\}$ is $\mu_{j}$-large,

3. for some $j$ the $\operatorname{set}\left\{x \in A: b_{j} \leq x\right\}$ is $\mu_{j}$-large.

Both nonobvious implications are proved by induction on $j$. They follow immediately from the definitions and are left to the reader.

Granted the claim we see that if $A$ is $\mu$-large and no $\mu_{j}=0$, then the construction cannot terminate. Indeed, let $\mu_{t}, b_{t}$ be its last item. Consider the sequence $\gamma_{0}=\mu_{t}$, $\gamma_{i+1}=\left\{\gamma_{i}\right\}\left(b_{t}\right)$ until we get a nonlimit $\gamma_{i}$. Then the set $\left\{x \in A: b_{i} \leq x\right\}$ is $\gamma_{i}$-large, where $\gamma_{i}>0$, so it has an element $>b_{t}$ and we have $b_{j+1}$ and $\mu_{j+1}$. But this is impossible, as the sequence $\mu_{j}$ would then be a decreasing sequence of ordinals.

If $A$ is $\mu$-small and $\mu_{j}=0$ for some $j$, then the set $\left\{x \in A: b_{j} \leq x\right\}$ being nonempty is 0 -large.

This proposition allows one to associate with every $a \in A$ an ordinal. That is, given a fixed $\mu$ such that $A$ is $\mu$-small (or at least $A \backslash\{\max (A)\}$ is $\mu$-small), we associate with every $a \in A$ the nonlimit $\mu_{j}$ such that $a=b_{j}$. But, of course, this assignment of ordinals to elements of $A$ depends on $\mu$. We shall write $\operatorname{KS}(\mu ; a)$ (or $\operatorname{KS}^{A}(\mu ; a)$ if necessary) for the last $\mu_{j}$ with $a=b_{j}$. Observe that for some $\mu$, $\mathrm{KS}(\mu ; a)$ is not defined for all elements of $A$, but they are defined if $A$ is at most $\mu$-large.

Let an ordinal $\mu$ and a set $A \subseteq \mathbb{N}$ be given, and assume that $A$ is $\mu$-small. Thus, $\operatorname{KS}(a)=\operatorname{KS}^{A}(\mu ; a)$ is defined for all $a \in A$ and is always greater than 0 . We need some simple observations concerning the sequence $\mu_{j}$ used in the definition of the association KS.

Lemma 2.8. For each $\mu$ and each $b>0$ we have $\operatorname{psn}(\{\mu\}(b)) \leq \max (b, \operatorname{psn}(\mu))$.

Proof. By induction on $\mu$. If $\mu=0$ the result is immediate. Also the successor step is obvious. If $\mu$ is of the form $\omega^{\tau+1}$, then $\{\mu\}(b)=\omega^{\tau} \cdot b, \operatorname{psn}(\mu)=\operatorname{psn}(\tau+1)$ and $\operatorname{psn}(\{\mu\}(b))=\operatorname{psn}\left(\omega^{\tau} \cdot b\right)=\max (\operatorname{psn}(\tau), b)$, so the result holds. If $\mu$ is of the form $\omega^{\tau}$ with limit $\tau$, then $\{\mu\}(b)=\omega^{\{\tau\}(b)}$, so the result follows from the inductive assumption. Finally, if $\mu$ has a nontrivial Cantor normal form expansion the result is immediate by the inductive assumption.

Corollary 2.9. For every set $A$ and every $\mu$ if $\mu_{j}$ and $b_{j}$ are as in the definition of the relation $\mathrm{KS}^{A}$, then $\operatorname{psn}\left(\mu_{j+1}\right) \leq \max \left(b_{j}, \operatorname{psn}\left(\mu_{j}\right)\right)$. In particular, if $\operatorname{psn}(\mu) \leq$ $\min (A)$, then for each $j$ we have $\operatorname{psn}\left(\mu_{j}\right) \leq b_{j}$.

We introduced the sequence $\omega_{m}$ above. We shall need the following generalization. We let $\omega_{m}(\alpha)$ be the sequence of ordinals defined by induction in the following manner: $\omega_{0}(\alpha)=\alpha, \omega_{m+1}(\alpha)=\omega^{\omega_{m}(\alpha)}$. Thus, $\omega_{0}(\omega)=\omega$, more generally $\omega_{m}(\omega)=\omega_{m+1}$ and $\omega_{m}(1)=\omega_{m}$ in this notation. The sequence $\omega_{m}(\alpha)$ is defined, in particular, for finite $\alpha$.

Proof of Theorem 2.6. Let $A \subset \mathbb{N}$ be $\omega_{k-1}$-small, i.e. $\omega_{k-2}\left(a_{0}\right)$-small, where, as usual, $a_{0}=\min (A)$. Let $\mu=\omega_{k-2}\left(a_{0}\right)$. Let $L_{k}$ be the partition constructed in Lemma 2.5. For $a=\left\langle a^{0}, \cdots, a^{k-1}\right\rangle$ we let

$$
L\left(a^{0}, \cdots, a^{k-1}\right)=L_{k}\left(\operatorname{KS}\left(a^{0}\right), \cdots, \operatorname{KS}\left(a^{k-1}\right)\right),
$$


where $\operatorname{KS}(a)=\mathrm{KS}^{A}(\mu ; a)$. It is easy to check that $L$ cannot admit a homogeneous set $D$ with strictly more than $\min (D)+\frac{(k-2) \cdot(k-1)}{2}+1$ elements.

In section 4 we shall use the same idea to construct partitions. But we shall use Cantor normal form expansions to bases of the form $\omega_{m}$. In order to obtain information on how large homogeneous sets exist for these partitions, we need the so-called estimation lemma (cf. lemma 3.1), because the objects associated with elements of a homogeneous set will be ordinals, which may be a limit, rather than directly natural numbers as above.

\section{The estimation Lemma}

If $\alpha, \beta$ are ordinals below $\varepsilon_{0}$ we define their natural sum (cf. [15]) in the following manner. We write both $\alpha, \beta$ in their Cantor normal forms, and permute items of both of these expansions so that we obtain a nonincreasing sequence of exponents. Then we write this sequence as the Cantor normal form of some ordinal which we denote $\alpha(+) \beta$ (to be more precise we join items which have the same exponents by removing the coefficients behind parentheses).

We let $F:\left(<\varepsilon_{0}\right) \rightarrow\left(<\varepsilon_{0}\right)$ be the function such that $F(\alpha)=\alpha+\varrho$, where $\varrho$ is the order type of the set of limit ordinals which do not exceed $\alpha$. Formally we define the function $F$ by the following conditions:

1. $F(0)=0$,

2. $F(\alpha+1)=F(\alpha)+1$,

3. $\beta \gg \alpha \Rightarrow F(\beta+\alpha)=F(\beta)(+) F(\alpha)$,

4. $F\left(\omega^{n}\right)=\omega^{n}+\omega^{n-1}+\cdots+\omega^{0}$ for $n<\omega$,

5. $F\left(\omega^{\alpha}\right)=\omega^{\alpha} \cdot 2+1$ for $\alpha \geq \omega$.

The $(+)$ sign in condition 3 denotes the natural sum of ordinals. This is needed as we do not require $F(\beta) \gg F(\alpha)$, and, indeed, for the $F$ determined by these conditions it is not so. As a matter of fact, it is easy to see that $F(\alpha)$ is never a limit.

Clearly, these conditions determine exactly one function $F$ from $\left(<\varepsilon_{0}\right)$ to $\left(<\varepsilon_{0}\right)$. Let us write an explicit formula for $F$. We write

$$
\alpha=\omega^{\alpha_{0}} \cdot a_{0}+\cdots+\omega^{\alpha_{s}} \cdot a_{s}+\omega^{n} \cdot m_{n}+\cdots+\omega^{0} \cdot m_{0},
$$

where $\alpha>\alpha_{0}>\cdots>\alpha_{s} \geq \omega$ and $n<\omega$ (we allow some $m_{i}$ 's to be zero, moreover we write $\omega^{0}$ rather than 1 to increase readability). Then $F(\alpha)$ is equal to

$$
\begin{gathered}
\omega^{\alpha_{0}} \cdot 2 a_{0}+\cdots+\omega^{\alpha_{s}} \cdot 2 a_{s} \\
+\omega^{n} \cdot m_{n}+\omega^{n-1} \cdot\left(m_{n}+m_{n-1}\right)+\cdots+\omega^{0} \cdot\left(m_{n}+\cdots+m_{0}\right) \\
+\left(a_{0}+\cdots+a_{s}\right) .
\end{gathered}
$$

The following result will be the heart of the matter in section 4

Lemma 3.1 (The estimation lemma). For every $\alpha<\varepsilon_{0}$ we have: for every $A \subseteq \mathbb{N}$ with $\min (A)>0$, if there exists a strictly decreasing function $G: A \rightarrow(\leq \alpha)$ such that $\forall a \in A \operatorname{psn}(G(a)) \leq a$, then $A$ is at most $F(\alpha)$-large.

We still need several preparatory facts before giving the proof of this.

Let us recall that by a well-known result (the so-called lemma on compositions, up to our knowledge it is due to S. Wainer; see [2], lemma 7)

$$
\forall \alpha \forall \beta \gg \alpha h_{\beta+\alpha}=h_{\beta} \circ h_{\alpha} .
$$


The following lemmas give some information about compositions even if the assumption $\beta \gg \alpha$ is not satisfied. The first lemma is a generalization of the well-known fact that Hardy hierarchy grows much quicker than the so-called slow growing hierarchy.

Lemma 3.2. For all $\alpha$, all $\beta \geq \alpha$ and all $a \geq 1$

$$
\left\{h_{\omega^{\beta}+\omega^{\alpha}}(a) \downarrow \Rightarrow\left[h_{\omega^{\alpha}} \circ h_{\omega^{\beta}}(a) \downarrow \& h_{\omega^{\alpha}} \circ h_{\omega^{\beta}}(a) \leq h_{\omega^{\beta}} \circ h_{\omega^{\alpha}}(a)\right]\right\} .
$$

Proof. We fix $\alpha$ and proceed by induction on $\beta$. If $\beta=\alpha$, then the result follows from Wainer's result on compositions, i.e. (13). Assume the fact for $\beta$; we check it for $\beta+1$. So assume that $h_{\omega^{\beta+1}+\omega^{\alpha}}(a) \downarrow$, i.e. $h_{\omega^{\beta+1}}\left(h_{\omega^{\alpha}}(a)\right) \downarrow$, i.e. $h_{\omega^{\beta} \cdot h_{\omega^{\alpha}(a)}}\left(h_{\omega^{\alpha}}(a)\right) \downarrow$. But we have that $h_{\omega^{\alpha}}\left(h_{\omega^{\beta+1}}(a)\right)=h_{\omega^{\alpha}}\left(h_{\omega^{\beta} \cdot a}(a)\right)$ is less than or equal to $h_{\omega^{\beta}} \circ h_{\omega^{\alpha}} \circ h_{\omega^{\beta} \cdot(a-1)}(a)$ by the inductive assumption applied to $a^{\prime}=h_{\omega^{\beta} \cdot(a-1)}(a)$. We continue putting $h_{\omega^{\alpha}}$ inside and see that this expression cannot exceed $h_{\omega^{\beta} \cdot a}\left(h_{\omega^{\alpha}}(a)\right) \leq h_{\omega^{\beta} \cdot h_{\omega^{\alpha}}(a)}\left(h_{\omega^{\alpha}}(a)\right)=h_{\omega^{\beta+1}}\left(h_{\omega^{\alpha}}(a)\right)$.

Assume the fact for all $\beta^{\prime}<\beta$, where $\beta$ is the limit. Assume $h_{\omega^{\beta}}\left(h_{\omega^{\alpha}}(a)\right) \downarrow$,

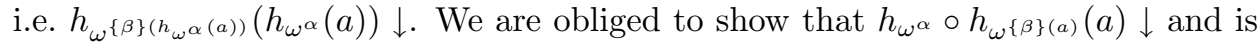
less than or equal to the element mentioned above. By the inductive assumption applied to $\beta^{\prime}=\{\beta\}(a)$ this element does not exceed $h_{\omega^{\{\beta\}(a)}}\left(h_{\omega^{\alpha}}(a)\right)$. By the results of Ketonen and Solovay [10] as collected in [2] (see lemma 4 in [2]), it suffices to show that $\omega^{\{\beta\}\left(h_{\omega^{\alpha}}(a)\right)} \Rightarrow{ }_{h_{\omega^{\alpha}}(a)} \omega^{\{\beta\}(a)}$. This is, again, a consequence of the results of Ketonen and Solovay; see [2], lemma 2, parts (v) and (iv).

Corollary 3.3. If $h_{\beta(+) \alpha}(a) \downarrow$, then $h_{\beta} \circ h_{\alpha}(a) \downarrow$ and $h_{\beta} \circ h_{\alpha}(a) \leq h_{\beta(+) \alpha}(a)$. In other words, if a set $A$ is $\beta(+) \alpha$-large, then there exists $u \in A$ such that $\{a \in A$ : $a \leq u\}$ is $\alpha$-large and $\{a \in A: u \leq a\}$ is $\beta$-large.

Proof. Let $\alpha$ and $\beta$ be given. Write $\beta(+) \alpha$ in its Cantor normal form:

$$
\omega^{\gamma_{s}} \cdot a_{s}+\cdots+\omega^{\gamma_{0}} \cdot a_{0}
$$

By the definition of $(+)$ and the usual + we may write $\beta+\alpha$ in the form

$$
\omega^{\gamma_{s}} \cdot b_{s}+\cdots+\omega^{\gamma_{0}} \cdot b_{0}
$$

where for all $i \leq s, b_{i} \leq a_{i}$. Let a set $A$ be given and let $x \in A$ be such that $h_{\beta(+) \alpha}^{A}(x) \downarrow$. By the lemma on compositions, i.e. (13), we infer that $h_{\beta(+) \alpha}^{A}(x)=$ $h_{\omega \gamma_{s} \cdot a_{s}}^{A} \circ \cdots \circ h_{\omega \gamma_{0} \cdot a_{0}}^{A}(x)$ and analogously for $h_{\beta+\alpha}^{A}(x)$, but with coefficients $b_{i}$ rather than $a_{i}$. By the lemma on compositions and the fact that each $h_{\delta}^{A}$ is increasing (this, again, is the standard fact about Hardy hierarchy, known from Ketonen and Solovay [10]; see [2], lemma 4), we may show by a simple induction on $i$ that for all $i \leq s, h_{\omega^{\gamma_{i} \cdot b_{i}}}^{A} \circ \cdots \circ h_{\omega \gamma_{0} \cdot b_{0}}^{A}(x) \downarrow$ and does not exceed $h_{\omega^{\gamma_{i} \cdot a_{i}}}^{A} \circ \cdots \circ h_{\omega \gamma_{0} \cdot a_{0}}^{A}(x) \downarrow$.

The following is a useful restatement of this corollary.

Corollary 3.4. If $A$ is at most $\alpha$-large, $B$ is at most $\beta$-large and $\max (A)=$ $\min (B)$, then $A \cup B$ is at most $\beta(+) \alpha$-large.

Proof. Assume the contrary. Let $E=A \cup B$. Thus, $E \backslash\{\max (E)\}$ is $\beta(+) \alpha-$ large. By Corollary 3.3 there exists $u \in E$ such that $\{x \in E \backslash\{\max (E)\}: x \leq u\}$ is $\alpha$-large and $\{x \in E \backslash\{\max (E)\}: u \leq x\}$ is $\beta$-large. If $u<\max (A)$, then $\{x \in A: x \leq u\}$ is $\alpha$-large, so $A$ cannot be at most $\alpha$-large. Thus, $u \geq \max (A)$. But $B \backslash\{\max (B)\} \supseteq\{x \in E \backslash\{\max (E)\}: x \geq u\}$, so by lemma 5 part (iii) in 
[2], $B \backslash\{\max (B)\}$ is $\beta$-large. This contradicts the assumption that $B$ is at most $\beta$-large.

Observe that given $a$ and $\alpha$ the number of these $\beta<\alpha$ for which $\operatorname{psn}(\beta) \leq a$ is finite. This will be made precise in the proof of the following lemma.

Lemma 3.5. For every $\alpha<\varepsilon_{0}$ and every $a \in \mathbb{N} \backslash\{0\}$ the set $\{\beta<\alpha: \operatorname{psn}(\beta) \leq a\}$ is finite.

Proof. As a matter of fact we prove something much stronger (but well known). Let $B(m, a)=\left\{\beta<\omega_{m}: \operatorname{psn}(\beta) \leq a\right\}$. Then the cardinality of $B(m, a)=\operatorname{tow}_{m}(a+1)$, where the iterated exponentiation tow was defined before Theorem 1.4 . We proceed by induction on $m$. For $m=0$ the only element of $B(0, a)$ is 0 , so the result holds. Assume the result for $m$. Let $B(m, a)=\left\{\alpha_{0}, \cdots, \alpha_{r}\right\}$ in decreasing order. Every $\alpha<\omega_{m+1}$ may be identified with the sequence of its coefficients in the Cantor normal form (which is changed slightly by allowing some coefficients to be 0$)$ whose exponents are $\alpha_{0}, \cdots, \alpha_{r}$. It follows that the number of these $\alpha$ 's is $(a+1)^{\operatorname{Card}(B(m, a))}$, indeed, the coefficients are $\leq a$, so there are $a+1$ of them.

Let us identify the greatest ordinal $<\alpha$ whose pseudonorm is $\leq a$. We define the symbol $\operatorname{GO}(a, \alpha)$ for $a>0$ and $\alpha>0$ by induction on $\alpha$. We let $\operatorname{GO}(a, 1)=0$, $\mathrm{GO}(a, \omega)=a$. Other cases are as follows:

$$
\operatorname{GO}(a, \alpha+1)=\left\{\begin{array}{lll}
\alpha & \text { if } & \operatorname{psn}(\alpha) \leq a \\
\operatorname{GO}(a, \alpha) & \text { if } \operatorname{psn}(\alpha)>a
\end{array}\right.
$$

Before giving the general limit step we put

$$
\mathrm{GO}\left(a, \omega^{\nu}\right)=\omega^{\mathrm{GO}(a, \nu)} \cdot a+\mathrm{GO}\left(a, \omega^{\mathrm{GO}(a, \nu)}\right) .
$$

Finally, if $\alpha=\xi+\omega^{\nu}$ in short Cantor normal form and $\xi \neq 0$, then

$$
\operatorname{GO}(a, \alpha)=\left\{\begin{array}{lll}
\xi+\operatorname{GO}\left(a, \omega^{\nu}\right) & \text { if } & \operatorname{psn}(\xi) \leq a \\
\operatorname{GO}(a, \xi) & \text { if } \operatorname{psn}(\xi)>a .
\end{array}\right.
$$

It is easy to check (by induction on $\alpha$ ) that $\operatorname{GO}(a, \alpha)<\alpha$ and $\operatorname{psn}(\operatorname{GO}(a, \alpha)) \leq a$. In fact, $\operatorname{GO}(a, \alpha)$ is the greatest ordinal with these properties.

Lemma 3.6. For every $a>0$ and every $\alpha>0$ we have: for all $\gamma$ if $\gamma<\alpha$ and $\operatorname{psn}(\gamma) \leq a$, then $\gamma \leq \mathrm{GO}(a, \alpha)$.

Proof. By induction on $\alpha$. For $\alpha=1$ and for $\alpha=\omega$ the fact is obvious. Assume the lemma for $\alpha$. Let $\gamma<\alpha+1$ and $\operatorname{psn}(\gamma) \leq a$. If $\gamma<\alpha$, then $\gamma \leq \operatorname{GO}(a, \alpha) \leq$ $\operatorname{GO}(a, \alpha+1)$ by the inductive assumption. So let $\gamma=\alpha$. Then the first case of the definition applies as $\operatorname{psn}(\gamma) \leq a$, so $\gamma \leq \alpha=\operatorname{GO}(a, \alpha+1)$.

Let $\alpha=\omega^{\nu}$ with $\nu>1$ and assume that for each $\beta<\alpha$ the lemma holds. Let $\gamma<\omega^{\nu}$ and $\operatorname{psn}(\gamma) \leq a$. Write $\gamma=\omega^{\xi} \cdot g+\psi$ with $\omega^{\xi} \ggg \psi$. Then $\xi<\nu$, because $\gamma<\alpha$, so $\xi \leq \operatorname{GO}(a, \nu)$ because $\operatorname{psn}(\xi) \leq \operatorname{psn}(\gamma) \leq a$, so we may apply the inductive assumption to $\nu$. Also $g \leq a$ because $\operatorname{psn}(\gamma) \leq a$. Moreover $\operatorname{psn}(\psi) \leq \operatorname{psn}(\gamma) \leq a$ and $\psi<\omega^{\xi}$, so $\psi<\omega^{\mathrm{GO}(a, \nu)}$, so by the inductive assumption applied to this ordinal, $\psi \leq \mathrm{GO}\left(a, \omega^{\mathrm{GO}(a, \nu)}\right)$ as required.

Let $\alpha=\xi+\omega^{\nu}$, where $\xi \gg \omega^{\nu}$ and $\xi \neq 0$. Let $\gamma$ satisfy the assumption. If $\gamma<\xi$, then $\gamma \leq \operatorname{GO}(a, \xi)$ by the inductive assumption applied to $\xi$, and in both cases in the definition $\operatorname{GO}(a, \xi) \leq \operatorname{GO}(a, \alpha)$. If $\gamma \geq \xi$, then $\gamma=\xi+\eta$ for some $\eta \ll \xi$ because $\xi \leq \gamma<\xi+\omega^{\nu}$. Also $\operatorname{psn}(\xi) \leq \operatorname{psn}(\gamma) \leq a$, hence 
$\mathrm{GO}(a, \alpha)=\xi+\mathrm{GO}\left(a, \omega^{\nu}\right)$. By the inductive assumption applied to $\omega^{\nu}$, we infer $\eta \leq \mathrm{GO}\left(a, \omega^{\nu}\right)$, so $\gamma=\xi+\eta \leq \xi+\mathrm{GO}\left(a, \omega^{\nu}\right)=\mathrm{GO}(a, \alpha)$.

Observe that in many cases $\mathrm{GO}(a, \lambda)>\{\lambda\}(a)$. In particular, the relation $\omega^{\lambda} \Rightarrow_{a} F\left(\operatorname{GO}\left(a, \omega^{\lambda}\right)\right)$ cannot hold. In the proof of the estimation lemma we shall need information of the following sort: if $z$ is large enough with respect to $a$, then $\omega^{\lambda} \Rightarrow_{z} F\left(\operatorname{GO}\left(a, \omega^{\lambda}\right)\right)$ holds. Our immediate goal is to make this precise. But at first we shall get some information about $\operatorname{GO}\left(a, \omega_{n}\right)$. Let $n, a>0$ and let

$$
\operatorname{GO}\left(a, \omega_{n}\right)=\omega^{\delta_{0}} \cdot d_{0}+\cdots+\omega^{\delta_{s}} \cdot d_{s}
$$

in the Cantor normal form (6).

Lemma 3.7. 1. The coefficients of the expansion of $\operatorname{GO}\left(a, \omega_{n}\right)$ are $d_{0}=d_{1}=$ $\cdots=d_{s}=a, \operatorname{GO}\left(a, \omega_{n}\right)$ is the nonlimit (i.e. $\delta_{s}=0$ ) and the length of this expansion is $s+1=\operatorname{tow}_{n-1}(a+1)$.

2. For $n>1$ the pseudonorm of $F\left(\operatorname{GO}\left(a, \omega_{n}\right)\right)$ is $\operatorname{psn}\left(F\left(\operatorname{GO}\left(a, \omega_{n}\right)\right)\right)=a$. tow $_{n-1}(a+1)$. Moreover, if $F\left(\operatorname{GO}\left(a, \omega_{n}\right)\right)=\tau+k$ with limit $\tau$ and integer $k$, then $\operatorname{psn}\left(F\left(\mathrm{GO}\left(a, \omega_{n}\right)\right)\right)=k$ and $\operatorname{psn}(\tau)=a^{2}$.

Proof. The only nonobvious fact from the first part is the information about the length of the expansion. Consider the expansion of $\operatorname{GO}\left(a, \omega_{n}\right)$. We assert that every ordinal $\varrho<\omega_{n-1}$ with $\operatorname{psn}(\varrho) \leq a$ occurs as the exponent in this expansion. For otherwise we could write $\operatorname{GO}\left(a, \omega_{n}\right)$ as $\beta+\gamma$, where $\beta \ggg \omega^{\varrho} \ggg \gamma$ and the ordinal $\beta+\omega^{\varrho} \cdot a+\gamma$ would be greater than $\operatorname{GO}\left(a, \omega_{n}\right)$ and still have pseudonorm $\leq a$, which is impossible. Thus the length $s+1$ of this expansion is just the number of the exponents, $\operatorname{Card}(B(n-1, a))=$ tow $_{n-1}(a+1)$; see the proof of Lemma 3.5.

The second part of the lemma is a consequence of the explicit formula for $F$, i.e. (12). If $\operatorname{GO}\left(a, \omega_{m}\right)$ is written in its Cantor normal form (14), then each summand $\omega^{\varrho} \cdot a$ added $a$ to the coefficient of $\omega^{0}$. But there are $\operatorname{tow}_{n-1}(a+1)$ such items of the expansion, so the claim about $k$ follows from the first part. Also, if $\varrho \geq \omega$, then the coefficient is multiplied by 2 , and if $\varrho<\omega$, then this coefficient is not changed, but the summand $\omega^{\varrho-1} \cdot a+\cdots+\omega^{0} \cdot a$ is added, so the result about $\tau$ follows from high school algebra (the theory of arithmetical progressions). We leave the details to the reader.

Corollary 3.8. Let $\alpha \leq \omega_{n}$ satisfy $\operatorname{psn}(\alpha) \leq a$. Then $\operatorname{psn}(F(\operatorname{GO}(a, \alpha))) \leq a$. tow $_{n-1}(a+1)$.

Proof. This follows from the discussion in the proof of Lemma 3.7. The reason is that if $\alpha$ satisfies the assumption, then all items of its Cantor normal form expansion have exponents which occur in items of the expansion of $\operatorname{GO}\left(a, \omega_{n}\right)$ and the coefficients of the expansion of $\alpha$ cannot exceed those of the expansion of $\mathrm{GO}\left(a, \omega_{n}\right)$.

The following corollary gives the promised information about $z$ such that $\omega^{\lambda} \Rightarrow_{z}$ $F\left(\operatorname{GO}\left(a, \omega^{\lambda}\right)\right)$.

Corollary 3.9. If $\lambda$ is such that $\operatorname{psn}(\lambda) \leq a$ and $\omega^{\lambda} \leq \omega_{n}$, then for every $z>$ $a \cdot \operatorname{tow}_{n-1}(a+1)$ we have $\omega^{\lambda} \Rightarrow_{z} F\left(\operatorname{GO}\left(a, \omega^{\lambda}\right)\right)$.

Proof. Immediate by Corollary 3.8 and Lemma 2.1

As a matter of fact, it will be convenient to prove the version of the estimation lemma with parameter. It is as follows. 
Lemma 3.10. For all $\alpha$, for all $\beta \gg \alpha$, and for all $A$ and $G$ if $G: A \rightarrow(\leq \beta+\alpha)$ is strictly decreasing, $\min (A)>0$ and $\forall x \in A \operatorname{psn}(G(x)) \leq x$, then setting $w=$ $\max x \in A: G(x) \geq \beta$ we have: $\{x \in A: x \leq w\}$ is at most $F(\alpha)$-large.

Lemma 3.10 implies the estimation lemma immediately ( $\operatorname{set} \beta=0$ ).

Proof of Lemma 3.10. Let $T(\alpha)$ denote the statement in Lemma 3.10 (without the quantifier $\forall \alpha$ ), and we prove $\forall \alpha T(\alpha)$ by induction on $\alpha$. For $\alpha=0$ there is nothing to prove, for the set $\{x \in A: G(x) \geq \beta\}$ is either void or has only one element, so is at most 0 -large. But $F(0)=0$.

Assume Lemma 3.10 for $\alpha$; we prove it for $\alpha+1$. So let $\beta \gg \alpha+1$ and let $G: A \rightarrow(\leq \beta+\alpha+1)$ satisfy the assumption. Let $A=\left\{a_{0}, \cdots, a_{r-1}\right\}$ in increasing order. Thus, $G\left(a_{0}\right) \leq \beta+\alpha+1$, hence $G\left(a_{1}\right) \leq \beta+\alpha$. We apply the inductive assumption to $G 1 A^{\prime}$, where $A^{\prime}=A \backslash\left\{a_{0}\right\}$. Hence $\left\{x \in A^{\prime}: G(x) \geq \beta\right\}$ is at most $F(\alpha)$-large. It follows that $\{x \in A: G(x) \geq \beta\}$ is at most $F(\alpha)+1$-large. But $F(\alpha+1)=F(\alpha)+1$, so we are done.

Let $\lambda$ be the limit and assume $\forall \alpha<\lambda T(\alpha)$. We consider several cases because the definition of $F$ depends on the form of $\lambda$. In each case let $G: A \rightarrow(\leq \beta+\lambda)$ satisfy the assumption, let $D=\{x \in A: G(x) \geq \beta\}$ be enumerated in increasing order, $D=\left\{d_{0}, \cdots, d_{r-1}\right\}$, and let $E=D \backslash\{\max (D)\}$.

CASE 1. $\lambda=\omega$. So let $\beta \gg \omega$ and let $G: A \rightarrow(\leq \beta+\omega)$ satisfy the assumption. Let $D=\{x \in A: G(x) \geq \beta\}$ as above. Then $G\left(d_{0}\right) \leq \beta+\omega$, so $G\left(d_{1}\right) \leq \beta+d_{1}$. Let $D^{\prime}=D \backslash\left\{d_{0}\right\}$. Then for $x \in D^{\prime}, G(x)$ must be of the form $G(x)=\beta+k_{x}$. The function $x \mapsto k_{x}$ being strictly decreasing is one-to-one, so $\operatorname{Card}\left(D^{\prime}\right) \leq d_{1}+1$. In other words, $D^{\prime}$ is at most $\omega$-large, hence $D$ is at most $\omega+1$-large. But $F(\omega)=\omega+1$.

Case 2. $\lambda=\omega^{n}$ for some $1<n<\omega$. Then $G\left(a_{0}\right) \leq \beta+\omega^{n}$, so

$$
G\left(a_{1}\right) \leq \mathrm{GO}\left(a_{1}, \beta+\omega^{n}\right) \leq \beta+\mathrm{GO}\left(a_{1}, \omega^{n}\right) \leq \beta+\omega^{n-1} \cdot a_{1}+\cdots+\omega^{0} \cdot a_{1} .
$$

By the inductive assumption applied to $\alpha=\omega^{n-1} \cdot a_{1}+\cdots+\omega^{0} \cdot a_{1}$ the set $D \backslash\left\{a_{0}\right\}$ is at most $F(\alpha)$-large. We also have $F(\alpha)=\omega^{n-1} \cdot a_{1}+\omega^{n-2} \cdot 2 a_{1}+\cdots+\omega^{1} \cdot(n-1) a_{1}+$ $\omega^{0} \cdot n a_{1}$. Assume that $D$ is not at most $F\left(\omega^{n}\right)$-large, so the set $E=D \backslash\{\max (D)\}$ is still $F\left(\omega^{n}\right)$-large. But $F\left(\omega^{n}\right)=\omega^{n}+\omega^{n-1}+\cdots+\omega^{0}$. Let $z=h_{\omega^{n-1}+\cdots+\omega^{0}}^{E}\left(a_{0}\right)$. It is easy to check that $z \geq n \cdot a_{1}$ (and the equality may hold only if $n=2$ ). From this we infer that $\omega^{n} \Rightarrow_{z} F(\alpha)$, also in the case $n=2$. Thus we see that $h_{\omega^{n}}^{E}(z) \downarrow$, so $h_{F(\alpha)}^{E}(z) \downarrow$, hence $h_{F(\alpha)}^{E}\left(a_{1}\right) \downarrow$. This contradicts the fact that $D \backslash\left\{a_{0}\right\}$ is at most $F(\alpha)$-large.

CASE 3. $\lambda=\omega^{\omega}$. As usual, we have $G\left(a_{0}\right) \leq \beta+\omega^{\omega}$, so

$$
G\left(a_{1}\right) \leq \beta+\operatorname{GO}\left(a_{1}, \omega^{\omega}\right)=\beta+\omega^{a_{1}} \cdot a_{1}+\omega^{a_{1}-1} \cdot a_{1}+\cdots+\omega^{0} \cdot a_{1} .
$$

By the inductive assumption applied to $\alpha=\omega^{a_{1}} \cdot a_{1}+\cdots+\omega^{0} \cdot a_{1}$ we infer that $D \backslash\left\{a_{0}\right\}$ is at most $F(\alpha)$-large, i.e., at most $\omega^{a_{1}} \cdot a_{1}+\omega^{a_{1}-1} \cdot 2 a_{1}+\cdots+\omega^{0} \cdot\left(a_{1}+1\right) \cdot a_{1}$-large. Assume that $D$ is not at most $F\left(\omega^{\omega}\right)$-large, so $D \backslash\left\{a_{0}\right\}$ is not at most $\omega^{\omega} \cdot 2$-large. Let $E=D \backslash\left\{a_{0}, \max (D)\right\}$ and $z=h_{\omega^{\omega}}^{E}\left(a_{1}\right)$. Then $z>\left(a_{1}+1\right) \cdot a_{1}$, so $\omega^{\omega} \Rightarrow_{z} F(\alpha)$. From this we infer that $h_{F(\alpha)}^{E}(z) \downarrow$ because $h_{\omega^{\omega}}^{E}(z) \downarrow$, hence $h_{F(\alpha)}^{E}\left(a_{1}\right) \downarrow$, so $D \backslash\left\{a_{0}\right\}$ is not at most $F(\alpha)$-large, a contradiction.

CASE 4. $\lambda=\omega^{\nu}$ with $\nu>\omega$. Using the same notation as above we see that $G\left(a_{1}\right) \leq \beta+\operatorname{GO}\left(a_{1}, \omega^{\nu}\right)$. We apply the inductive assumption to $\alpha=\operatorname{GO}\left(a_{1}, \omega^{\nu}\right)$ and infer that $D \backslash\left\{a_{0}\right\}$ is at most $F\left(\operatorname{GO}\left(a_{1}, \omega^{\nu}\right)\right)$-large. Assume that $D$ is not at most $F\left(\omega^{\nu}\right)$-large, so is not $\omega^{\nu} \cdot 2+1$-large. Then $D \backslash\left\{a_{0}\right\}$ is not at most 
$\omega^{\nu} \cdot 2$-large, so $E=D \backslash\left\{a_{0}, \max (D)\right\}$ is $\omega^{\nu} \cdot 2$-large. Let $z=h_{\omega^{\nu}}^{E}\left(a_{1}\right)$. Obviously, $z>\operatorname{psn}\left(F\left(\operatorname{GO}\left(a_{1}, \omega^{\nu}\right)\right)\right)$, so $\omega^{\nu} \Rightarrow_{z} F\left(\operatorname{GO}\left(a_{1}, \omega^{\nu}\right)\right)$, hence $h_{F\left(\operatorname{GO}\left(a_{1}, \omega^{\nu}\right)\right)}^{E}(z) \downarrow$. From this we infer that $h_{F\left(\mathrm{GO}\left(a_{1}, \omega^{\nu}\right)\right)}^{E}\left(a_{1}\right) \downarrow$, and we get a contradiction with the fact that $D \backslash\left\{a_{0}\right\}$ is at most $F\left(\mathrm{GO}\left(a_{1}, \omega^{\nu}\right)\right)$-large.

CASE 5. The Cantor normal of $\lambda$ is nontrivial, that is, if $\lambda$ is written in its Cantor normal form (6), then there are at least two summands or the coefficient is strictly greater than 1 . Then $\lambda=\gamma+\delta$ for some $\gamma \gg \delta$ with $\gamma, \delta \neq 0$. Let $\beta, G, A$ satisfy the assumptions. Then the set $\{x \in A: G(x) \geq \beta+\gamma\}$ is at most $F(\delta)$-large, indeed, every $G(x)$ for $x$ in it must be of the form $G(x)=\beta+\gamma+\tau_{x}$, and the function $x \rightarrow \tau_{x}$ satisfies the inductive assumption for $\delta<\lambda$. Similarly, the set $\{x \in A: \beta+\gamma \geq G(x) \geq \beta\}$ is at most $F(\gamma)$-large. Their union is just $\{x \in A: G(x) \geq \beta\}$. It is at most $F(\gamma)(+) F(\delta)$-large by Corollary 3.3. But $F(\gamma)(+) F(\delta)=F(\gamma+\delta)$ by the definition of $F$.

The estimation lemma would become false if we require $G$ to be increasing rather than decreasing. Indeed, let $A$ be any subset of $\mathbb{N}$ and let $H$ be the identity function. Then $H: A \rightarrow(<\omega)$ satisfies the second assumption. But $A$ is arbitrary, so it need not be at most $F(\omega)$-large.

\section{The MAIN RESUlT}

In this section we use the same idea as in sections 1 and 2, But this time we shall use expansions of ordinals to other bases than $\omega$, in fact, our bases will be just $\omega_{n}$. In fact, in this section we work with bases $\omega_{n}$ and $\omega_{m}$ for $m, n>1$ because the case $\omega$ was considered in section 2, so from now on expansions to the base $\omega$ will be used merely as a technical tool.

The reader will find benefits from reading sections 7.5-7.7 in [15] to learn more about expansions to other bases. In particular, the reader is supposed to know that every $\alpha<\varepsilon_{0}$ may be written in the Cantor normal form to the base $\omega_{n}$

$$
\alpha=\left(\omega_{n}\right)^{\alpha_{0}} \cdot \beta_{0}+\cdots+\left(\omega_{n}\right)^{\alpha_{s}} \cdot \beta_{s}
$$

for some $\alpha_{0}>\alpha_{1}>\cdots>\alpha_{s}$ with $\alpha>\alpha_{0}$ and $\beta_{0}, \cdots, \beta_{s}<\omega_{n}$. Moreover, this expansion is uniquely determined by $\alpha$ and $n$. The next fact we need is as follows. Let $\alpha$ and $\gamma$ both be written in their Cantor normal forms (with the same $n$ ). Then the usual ordering between $\alpha$ and $\gamma$ is the same as the one defined by these expansions (one compares the leftmost exponents in these expansions, if they are different, then the same inequality holds between $\alpha$ and $\gamma$; if these exponents are the same, then one compares the coefficients, etc.). Simply such an ordering (defined using expansions to the base $\omega_{n}$ ) is the same as the one obtained by the relation of embeddability onto an initial segment of sets of the appropriate order types.

The next information which will be needed below is as follows. The notion of pseudonorm of an ordinal was defined using expansions to the base $\omega$.

Lemma 4.1. Let $n>0$ and $\alpha<\varepsilon_{0}$. Write $\alpha$ to the base $\omega_{n}$ (i.e. in the form (15) above). Then $\operatorname{psn}(\alpha) \geq \max \left(\operatorname{psn}\left(\alpha_{0}\right), \cdots, \operatorname{psn}\left(\alpha_{s}\right), \operatorname{psn}\left(\beta_{0}\right), \cdots, \operatorname{psn}\left(\beta_{s}\right)\right)$.

Proof. If $n=1$ the fact is obvious, so let $n \geq 2$. Below (if $n=2$ ) we write $\omega_{0}=1$ for uniformity of the argument. Consider a single summand in the expansion of $\alpha$ to the base $\omega_{n}$. It is of the form $\left(\omega_{n}\right)^{\gamma} \cdot \delta$, i.e. $\omega^{\omega_{n-1} \cdot \gamma} \cdot \delta$. Let $\gamma=\omega^{\gamma_{0}} \cdot g_{0}+\cdots+\omega^{\gamma_{r}} \cdot g_{r}$ be the expansion of $\gamma$ to the usual base $\omega$ and let $\delta=\omega^{\delta_{0}} \cdot d_{0}+\cdots+\omega^{\delta_{t}} \cdot d_{t}$ be 
the Cantor expansion of $\delta$ to the same base. Thus our summand $\left(\omega_{n}\right)^{\gamma} \cdot \delta$ may be written as

$$
\begin{gathered}
\left(\omega_{n}\right)^{\omega^{\gamma_{0}} \cdot g_{0}+\cdots+\omega^{\gamma_{r}} \cdot g_{r}} \cdot\left(\omega^{\delta_{0}} \cdot d_{0}+\cdots+\omega^{\delta_{t}} \cdot d_{t}\right) \\
=\omega^{\omega_{n-1} \cdot\left(\omega^{\gamma_{0}} \cdot g_{0}+\cdots+\omega^{\gamma_{r}} \cdot g_{r}\right)} \cdot\left(\omega^{\delta_{0}} \cdot d_{0}+\cdots+\omega^{\delta_{t}} \cdot d_{t}\right) \\
=\omega^{\omega^{\omega_{n-2}+\gamma_{0}} \cdot g_{0}+\cdots+\omega^{\omega_{n-2}+\gamma_{r}} \cdot g_{r}} \cdot\left(\omega^{\delta_{0}} \cdot d_{0}+\cdots+\omega^{\delta_{t}} \cdot d_{t}\right) .
\end{gathered}
$$

Because of the sum in parentheses this expression is, again, a sum. Let us consider a single summand of this expression. It corresponds to the the item $\omega^{\delta_{j}} \cdot d_{j}$ and is of the form

$$
\omega^{\omega^{\omega_{n-2}+\gamma_{0}} \cdot g_{0}+\cdots+\omega^{\omega_{n-2}+\gamma_{r}} \cdot g_{r}+\delta_{j}} \cdot d_{j} .
$$

Observe that $\delta_{j}<\omega_{n-1}$, indeed, $\omega^{\delta}<\omega_{n}$ as a coefficient in the expansion to the base $\omega_{n}$, so because of the presence of $\omega_{n-2}$ at the second level of exponents, the first level of exponents is written in the normal form, so none of its items is absorbed by the next one. The expansion of the summand under consideration is to the base $\omega$, so we can see its pseudonorm directly. Thus we see that $\operatorname{psn}(\alpha) \geq$ $\operatorname{psn}\left(\left(\omega_{n}\right)^{\gamma} \cdot \delta\right) \geq \operatorname{psn}(\gamma)$, indeed, all exponents and coefficients of the expansion of $\gamma$ occur in this expansion at the first and second level of exponents. Also, $\operatorname{psn}(\alpha) \geq \operatorname{psn}\left(\delta_{j}\right)$ for $j \leq t$ because each $\delta_{j}$ occurs in this expansion at the first level of exponents. Moreover, $\operatorname{psn}(\alpha) \geq d_{j}$ for all $j$, as is seen from this expansion, so together $\operatorname{psn}(\alpha) \geq \operatorname{psn}(\delta)$. The next observation to be used in the sequel is

$$
\text { if } 1 \leq k \leq m \text {, then }\left(\omega_{k}\right)^{\omega_{m}}=\omega_{m+1} .
$$

The result is obvious for $k=1$, so assume that $k \geq 2$. Then

$$
\left(\omega_{k}\right)^{\omega_{m}}=\left(\omega^{\omega_{k-1}}\right)^{\omega_{m}}=\omega^{\omega_{k-1} \cdot \omega_{m}}=\omega^{\omega^{\omega_{k-2}+\omega_{m-1}}}=\omega^{\omega^{\omega_{m-1}}}=\omega_{m+1}
$$

as desired.

We continue the convention from section 2 Thus, we write sets of ordinals in decreasing order. Also, if $\alpha=\left(\alpha_{0}, \cdots, \alpha_{s}\right)$ is a tuple of ordinals (e.g. $\alpha$ is the argument of a partition), it is tacitly assumed that $\alpha_{0}>\cdots>\alpha_{s}$, unless explicitly stated otherwise.

Let $m>1$ be fixed. Following the idea of section 2 we construct a sequence of partitions of sets of ordinals. For $\alpha<\varepsilon_{0}$ we let $v\left(\omega_{m} ; \alpha, \delta\right)$ be the coefficient at $\left(\omega_{m}\right)^{\delta}$ in the normal form expansion of $\alpha$ to the base $\omega_{m}$. Thus, using the notation from (15) with $m$ instead of $n, v\left(\omega_{m}, \alpha, \alpha_{i}\right)=\beta_{i}$ and $v\left(\omega_{m} ; \alpha, \delta\right)=0$ for $\delta$ not of the form $\alpha_{i}$. This allows us to define

$$
\mathrm{LD}\left(\omega_{m} ; \alpha, \beta\right)=\max \delta: v\left(\omega_{m} ; \alpha, \delta\right) \neq v\left(\omega_{m} ; \beta, \delta\right) .
$$

The main property of this partition is as follows.

Lemma 4.2. Let $\Gamma$ be a set of ordinals below $\varepsilon_{0}$ which is homogeneous with respect to $\operatorname{LD}\left(\omega_{m}\right)$. Then there exists an increasing function $\Theta: \Gamma \rightarrow\left(<\omega_{m}\right)$ such that for all $\gamma \in \Gamma, \operatorname{psn}(\Theta(\gamma)) \leq \operatorname{psn}(\gamma)$.

Proof. Let $\Gamma$ satisfy the assumption. Thus, there exists $\delta$ such that for all $\alpha, \beta \in \Gamma$, $\mathrm{LD}\left(\omega_{m} ; \alpha, \beta\right)=\delta$. It follows that every $\alpha \in \Gamma$ may be written as $\varrho+\left(\omega_{m}\right)^{\delta} \cdot \xi_{\alpha}+\tau_{\alpha}$ to the base $\omega_{m}$, where all exponents in $\varrho$ are strictly greater than $\delta$ and all exponents in $\tau_{\alpha}$ are strictly smaller than $\delta$. Clearly, $\varrho$ does not depend on $\alpha$ and the function $\Theta(\alpha)=\xi_{\alpha}$ has the required property by Lemma 4.1, and the remark on the ordering of ordinals given by their expansions to the bases of the form $\omega_{m}$. 
The partitions of $\left[<\varepsilon_{0}\right]^{j}$ to be constructed below will have the property (roughly speaking) that every homogeneous set will carry a function as above. Let us introduce the appropriate terminology concerning functions. Let $A \subset \mathbb{N}$. A function $G: A \rightarrow\left(<\omega_{m}\right)$ will be called an estimating function (or a function $\omega_{m}{ }^{-}$ estimating $A$ if necessary) if it satisfies the assumptions of the estimation lemma, i.e. it is strictly decreasing and for all $a \in A, \operatorname{psn}(G(a)) \leq a$. If $\Gamma \subseteq\left(<\varepsilon_{0}\right)$ and $\Theta: \Gamma \rightarrow\left(<\omega_{m}\right)$, we say that $\Theta$ is an ordinal estimating function (or a function $\omega_{m}$-ordinal estimating $\Gamma$ ) if it is strictly increasing and for all $\gamma \in \Gamma$, $\operatorname{psn}(\Theta(\gamma)) \leq \operatorname{psn}(\gamma)$. Of course, we shall be interested mainly in estimating functions, and we shall obtain them from the ordinal estimating functions by means of $\mathrm{KS}$.

The following is the analogue of the partition KE (cf. (9)):

$$
\operatorname{KE}\left(\omega_{m} ; \alpha, \beta, \gamma\right)=\left\{\begin{array}{lll}
0 & \text { if } & \operatorname{LD}\left(\omega_{m} ; \alpha, \beta\right)<\operatorname{LD}\left(\omega_{m} ; \beta, \gamma\right), \\
1 & \text { if } \operatorname{LD}\left(\omega_{m} ; \alpha, \beta\right)=\operatorname{LD}\left(\omega_{m} ; \beta, \gamma\right), \\
2 & \text { if } \operatorname{LD}\left(\omega_{m} ; \alpha, \beta\right)>\operatorname{LD}\left(\omega_{m} ; \beta, \gamma\right) .
\end{array}\right.
$$

Once again, this is a partition of $\left[\varepsilon_{0}\right]^{3}$ into 3 parts.

Lemma 4.3. (i) If $\Gamma$ is a finite subset of $\left(<\varepsilon_{0}\right)$ is such that $\mathrm{KE}\left(\omega_{m}\right)$ colors $[\Gamma]^{3}$ by 1 , then there exists an ordinal estimating function $\Theta$ defined on $\Gamma$ with values in $\left(<\omega_{m}\right)$. (ii) If $\Gamma$ is a finite subset of $\left(<\varepsilon_{0}\right)$ is such that $\max (\Gamma)<\omega_{m+s}$ and $\mathrm{KE}\left(\omega_{m}\right)$ colors $[\Gamma]^{3}$ by 2 , then there exists an ordinal estimating function $\Theta$ defined on $\Gamma \backslash\{\min (\Gamma)\}$ with values in $\left(<\omega_{m+s-1}\right)$. (iii) If $\Gamma$ is a finite subset of $\left(<\varepsilon_{0}\right)$ such that $\max (\Gamma)<\omega_{m+s}$, and $\mathrm{KE}\left(\omega_{m}\right)$ colors $[\Gamma]^{3}$ by 0 , then $\operatorname{Card}(\Gamma) \leq$ tow $_{m+s-1}(\operatorname{psn}(\max (\Gamma))+1)+1$.

Proof. Let $\Gamma=\left\{\gamma_{0}, \cdots, \gamma_{r-1}\right\}$ be a decreasing enumeration of $\Gamma$. Assume first that $[\Gamma]^{3}$ has color 1 . We write each $\gamma_{i}$ in the Cantor normal form to the base $\omega_{m}$ and see that there exists $\delta$ such that $\gamma_{i}=\varrho+\left(\omega_{m}\right)^{\delta} \cdot \xi_{i}+\tau_{i}$. By the assumption of the case (neither $\varrho$ nor $\delta$ depends on $i$, and the function $\Theta\left(\gamma_{i}\right)=\xi_{i}$ has the required property) the nonobvious property follows from Lemma 4.1

Assume now that $[\Gamma]^{3}$ has color 2 . Let $\delta_{i}=\operatorname{LD}\left(\omega_{m} ; \gamma_{i}, \gamma_{i+1}\right)$. Then the function $\Theta\left(\gamma_{i}\right)=\delta_{i}$ has the required property. In particular, its values are smaller than $\omega_{m+s-1}$ by $(16)$.

Finally, let $[\Gamma]^{3}$ have color 0 . We let $\delta_{i}$ be as above. We assert that $\left(\omega_{m}\right)^{\delta_{i}}$ occurs with a nonzero coefficient in the Cantor normal form expansion to the base $\omega_{m}$ of $\gamma_{0}$ for every $i<r-1$. Indeed, fix $i<r-1$. We write $\gamma_{i}=\varrho+\left(\omega_{m}\right)^{\delta_{i}} \cdot \xi_{i}+\tau_{i}$ and compare this with the expansion of $\gamma_{i+1}$ to the base $\omega_{m}$. We see that $\xi_{i}$ must be greater than the coefficient at $\left(\omega_{m}\right)^{\delta_{i}}$ in the expansion of $\gamma_{i+1}$. In particular, $\xi_{i}>0$. It follows that the set $\Delta=\left\{\delta_{i}: i<r-1\right\}$ is of cardinality at most tow $_{m+s-1}\left(\operatorname{psn}\left(\gamma_{0}\right)+1\right)$ by the argument of Lemma 3.5 .

We shall also write $L_{3}\left(\omega_{m} ; \alpha, \beta, \gamma\right)=\mathrm{KE}\left(\omega_{m} ; \alpha, \beta, \gamma\right)$ for uniformity of further notation.

Lemma 4.4. Let $m>1$. Then for every $k \geq 3$ there exists a partition $L_{k}\left(\omega_{m}\right)$ of $\left[\varepsilon_{0}\right]^{k}$ into $3^{k-2}$ parts such that for every $\Gamma \subseteq\left(<\varepsilon_{0}\right)$ homogeneous for $L_{k}\left(\omega_{m}\right)$, if $\max (\Gamma)<\omega_{m+k-2}$, then letting $\Gamma^{\prime}$ be $\Gamma$ without the last $\frac{(k-2)(k-1)}{2}$ elements we have: there exists an ordinal estimating function $\Theta: \Gamma^{\prime} \rightarrow\left(<\omega_{m}\right)$ or $\operatorname{Card}(\Gamma) \leq$ tow $_{m+k-3}(\operatorname{psn}(\max (\Gamma))+1)+k-2$. 
Proof. By induction on $k$, the result for $k=3$ was proved above. Assume the result for $k$; we construct the partition for $k+1$. Let $\alpha=\left(\alpha^{0}, \cdots, \alpha^{k}\right)$ be a $k+1-$ tuple of ordinals. We begin by putting $G(\alpha)=L_{3}\left(\omega_{m} ; \alpha^{0}, \alpha^{1}, \alpha^{2}\right)$, so this does not depend on the last $k-2$ coordinates of the sequence $\alpha$. As usual, we have: if $\Gamma$ is homogeneous for $G$, then $\Gamma^{\prime \prime}$ is homogeneous for $L_{3}\left(\omega_{m}\right)$, where $\Gamma^{\prime \prime} \operatorname{denotes} \Gamma$ without its last $k-2$ elements.

For $\alpha$ as above we let $\delta^{i}=\operatorname{LD}\left(\omega_{m} ; \alpha^{i}, \alpha^{i+1}\right)$. As previously, this $k$-tuple need not be monotonic. Let

$$
W(\alpha)= \begin{cases}L_{k}\left(\omega_{m} ; \delta^{0}, \cdots, \delta^{k-1}\right) & \text { if } \delta^{0}>\cdots>\delta^{k-1} \\ L_{k}\left(\omega_{m} ; \delta^{k-1}, \cdots, \delta^{0}\right) & \text { if } \delta^{0}<\cdots<\delta^{k-1} \\ 0 & \text { in other cases. }\end{cases}
$$

Finally we put $L_{k+1}\left(\omega_{m} ; \alpha\right)=\langle G(\alpha), W(\alpha)\rangle$. We assert that this partition has the required properties. So let $\Gamma=\left\{\gamma_{0}, \cdots, \gamma_{r-1}\right\}$ written in decreasing order be monochromatic for $L_{k+1}\left(\omega_{m}\right)$ and let $\Gamma^{\prime \prime}$ denote $\Gamma$ without its last (i.e. smallest) $k-2$ elements. Also let $\Gamma^{\prime}$ denote the set under consideration, i.e. $\Gamma$ without its last $\frac{k(k-1)}{2}$ elements.

CASE 1 . $G$ colors $[\Gamma]^{k+1}$ by 1 . Then $L_{3}\left(\omega_{m}\right)$ colors $\left[\Gamma^{\prime \prime}\right]^{3}$ by 1 , so by part (i) of Lemma 4.3 there exists a function ordinal $\omega_{m}$-estimating $\Gamma^{\prime \prime}$. The domain of this function contains the required set $\Gamma^{\prime}$.

CASE 2. $G$ colors $\left[\Gamma^{3}\right]$ by 2 . Then $L_{3}\left(\omega_{m}\right)$ colors $\left[\Gamma^{\prime \prime}\right]^{3}$ by 2 . Let $\delta_{i}=$ $\mathrm{LD}\left(\omega_{m} ; \gamma_{i}, \gamma_{i+1}\right)$ for $i<(r-1)-(k-2)$. By the assumption of the case, the set $\Delta=\left\{\delta_{0}, \cdots, \delta_{r-k}\right\}$ is written in decreasing order. By homogeneity of $\Gamma$ with respect to $W$, the set $\Delta$ is monochromatic with respect to $L_{k}\left(\omega_{m}\right)$. We distinguish two subcases.

Subcase (a). $\operatorname{Card}(\Delta) \leq$ tow $_{m-k-3}(\operatorname{psn}(\max (\Delta))+1)+k-2$. Obviously $\operatorname{psn}(\max (\Delta)) \leq \operatorname{psn}\left(\max \left(\Gamma^{\prime \prime}\right)\right) \leq \operatorname{psn}(\max (\Gamma))$, so

$$
\operatorname{Card}\left(\Gamma^{\prime \prime}\right) \leq \operatorname{tow}_{m+k-3}(\operatorname{psn}(\max (\Gamma))+1)+k-1,
$$

so $\operatorname{Card}(\Gamma)$ is smaller than or equal to

$$
\text { tow }_{m+k-3}(\operatorname{psn}(\max (\Gamma))+1)+2 k-3<\operatorname{tow}_{m+k-2}(\operatorname{psn}(\max (\Gamma))+1)+k-1,
$$

so the second case in the lemma holds.

Subcase (b). There exists a function $\Theta$ whose ordinal $\omega_{m}$-estimates $\Delta$ without its last $\frac{(k-2)(k-1)}{2}$ elements. Consider the function $\delta_{i} \mapsto \gamma_{i}=\Theta\left(\delta_{i}\right)$. This function ordinal $\omega_{m}$-estimates $\Gamma$ without its last $\frac{(k-2)(k-1)}{2}+1+k-2=\frac{k(k-1)}{2}$ elements as required.

CAse 3. $G$ colors $[\Gamma]^{3}$ by 0 . Then $L_{3}\left(\omega_{m}\right)$ gives $\left[\Gamma^{\prime \prime}\right]^{3}$ color 0 , so by part (iii) of Lemma $4.3 \operatorname{Card}\left(\Gamma^{\prime \prime}\right) \leq \operatorname{tow}_{m+k-2}\left(\operatorname{psn}\left(\max \left(\Gamma^{\prime \prime}\right)\right)+1\right)+1$. But obviously, $\max \left(\Gamma^{\prime \prime}\right)=\max (\Gamma)$, so $\operatorname{Card}(\Gamma) \leq \operatorname{tow}_{m+k-2}(\operatorname{psn}(\max (\Gamma))+1)+k-1$ as required.

Before applying Lemma 4.4 to partitions of sets of integers we show that in one of the cases considered above the homogeneous set is small indeed.

Lemma 4.5. Let $S$ denote the usual successor function, $S(x)=x+1$, and let $S_{\alpha}$ denote Hardy iterations of $S$. Then for all $m \geq 1$ we have $\forall x>0, S_{\omega^{2} \cdot 2 m}(x) \geq$ $\operatorname{tow}_{m}(x+1)$. 
Proof. By induction on $m$. If $m=1$, then obviously $S_{\omega^{2} \cdot 2}(x)=2^{2^{x} \cdot x} \cdot 2^{x} \cdot x>x+1$. Assume the result for $m$. Then

$$
\begin{gathered}
S_{\omega^{2} \cdot(2 m+2)}(x)=S_{\omega^{2} \cdot 2}\left(S_{\omega^{2} \cdot 2 m}(x)\right) \geq S_{\omega^{2}}\left(\operatorname{tow}_{m}(x+1)\right) \\
=2^{2^{\text {tow }_{m}(x+1)} \cdot \operatorname{tow}_{m}(x+1)} \cdot 2^{\text {tow }_{m}(x+1)} \cdot \operatorname{tow}_{m}(x+1) \geq 2^{\text {tow }(x+1)} \cdot \operatorname{tow}_{m}(x+1) \\
=\left(2^{\text {tow }_{m}(x+1)}\right)^{2^{\text {tow }_{m}(x+1)}} \geq(x+1)^{\text {tow }_{m}(x+1)}
\end{gathered}
$$

as required.

Lemma 4.6. Let $D \subseteq \mathbb{N}$ be such that $\operatorname{Card}(D)+d_{0} \leq S_{\alpha}\left(d_{0}\right)$ and $d_{0}=\min (D)>1$. Then $D$ is at most $\alpha$-large.

Proof. Consider the interval $B=\left[d_{0}, d_{0}+S_{\alpha}\left(d_{0}\right)\right]$ of $\mathbb{N}$. This set is exactly $\alpha$-large. Let $D^{\prime}=D \backslash\{\max (D)\}$. Assume that $D^{\prime}$ is $\alpha$-large. The initial segment $B^{\prime}$ of $B$ having the same cardinality as $D^{\prime}$ has consecutive elements less than or equal to consecutive elements of $D^{\prime}$, so is $\alpha$-large as well by lemma 5 in [2]. But this segment is of the form $\left[d_{0}, d_{0}+\operatorname{Card}(D)+d_{0}-1\right]$, so is included in $\left[d_{0}, d_{0}+S_{\alpha}\left(d_{0}\right)\right)$, and so is included in $B \backslash\{\max (B)\}$. This is impossible; indeed, $B$ is at most $\alpha$-large.

The following theorem and its corollary are the main results of this paper. They constitute a generalization of our version of the results of Ketonen and Solovay 10. as stated and proved above; see Theorem 2.6.

Theorem 4.7. Let $k \geq 3$ and $m>1$. Let $A \subseteq \mathbb{N}$ be at most $\omega_{m+k-2}$-large with $\min (A) \geq k$. Then there exists a partition $L_{k}:[A]^{k} \rightarrow 3^{k-2}$ such that every $D \subseteq A$ monochromatic for $L_{k}$ is at most $F\left(\omega_{m}\right)+\frac{(k-1)(k-2)}{2}$-large.

Proof. Let $A$ satisfy the assumption. Let $L_{k}\left(\omega_{m}\right)$ be a partition of $\left[\varepsilon_{0}\right]^{k}$ with the properties described in Lemma 4.4 For $a=\left(a^{0}, \cdots, a^{k-1}\right)$ we let

$$
R_{k}(a)=L_{k}\left(\omega_{m} ; \mathrm{KS}^{A}\left(\omega_{m+k-2} ; a^{0}\right), \cdots, \mathrm{KS}^{A}\left(\omega_{m+k-2} ; a^{k-1}\right)\right)
$$

and verify that this partition has the desired properties. So let $D$ be a subset of $A$ which is homogeneous for $R_{k}$. Then $\Gamma=\left\{\operatorname{KS}^{A}\left(\omega_{m+k-2} ; d\right): d \in D\right\}$ is homogeneous for $L_{k}\left(\omega_{m}\right)$. Let $\Gamma^{\prime}$ denote $\Gamma$ without its $\frac{(k-2)(k-1)}{2}$ smallest elements and let $D^{\prime}$ be $D$ without its last $\frac{(k-2)(k-1)}{2}$ elements.

CASE 1. There exists an ordinal estimating function $\Theta: \Gamma^{\prime} \rightarrow\left(<\omega_{m}\right)$. Then $D^{\prime}$ is at most $F\left(\omega_{m}\right)$-large by the estimation lemma (i.e. Lemma 3.1), and the result follows. (A minor point should be remarked here. The smallest elements of $\Gamma$ correspond to the greatest elements of $D$, thus to obtain the required conclusion we apply lemma 5 in [2].)

CASE 2. $\operatorname{Card}(\Gamma) \leq S_{\omega^{2}(2 m+2 k-4)}\left(d_{0}\right)$. Then $D$ has the same cardinality, so is $\omega^{\omega}$-small, so is $F\left(\omega_{m}\right)$-small as $m>1$.

We hope that the meaning of the relation $A \rightarrow(\alpha)_{m}^{r}$ is clear.

Corollary 4.8. Let $m, k \in \mathbb{N}$. Let $\alpha=F\left(\omega_{m}\right)+\frac{(k-2)(k-1)}{2}+1$. Let $A$ be such that $A \rightarrow(\alpha)_{3^{k-2}}^{k}$ and $3 \leq k \leq \min (A)$. Then $A$ is $\omega_{m+k-2}$-large.

Proof. At first we check by induction on $\alpha$ that for every $B$, if $B$ is $\alpha$-small, then there exists $C$ such that $\max (B)<\min (C)$ and $B \cup C$ is exactly $\alpha$-large. This is obvious if $B=\emptyset$, so from now on we assume that $B$ is nonvoid and begin induction from $\alpha=1$. If $\alpha=1$, then $B$ being 1 -small has at most one element, so each one element set $C=\{c\}$ satisfies our demand, of course provided $c>\min (B)$. Assume 
the result for $\alpha$ and let $B$ be $\alpha+1$-small. Then $B \backslash\{\min (B)\}$ is $\alpha$-small, so by the inductive assumption there exists $C$ with all elements greater than $\max (B)$ and such that $(B \backslash\{\min (B)\}) \cup C$ is exactly $\alpha$-large. Clearly, such $C$ has the desired property for $B$ and $\alpha+1$. Assume the conclusion for all $\alpha<\lambda$, where $\lambda$ is the limit. Let $B$ be $\lambda$-small. Then $B$ is $\{\lambda\}(\min (B))$-small, and we apply the inductive assumption to this set.

Granted the claim we argue as follows. Assume that $A$ is $\omega_{m+k-2}$-small. Let $C$ be as in the claim, so $\min (A)<\max (C)$ and $A \cup C$ is exactly $\omega_{m+k-2}$-large. By Corollary 4.8 there exists a partition $L$ of $[A \cup C]^{k}$ into $3^{k-2}$ parts without an

$F\left(\omega_{m}\right)+\frac{(k-2)(k-1)}{2}+1$-large monochromatic set. We restrict $L$ to $[A]^{k}$ and see that this restriction does not admit an $F\left(\omega_{m}\right)+\frac{(k-2)(k-1)}{2}+1$-large homogeneous set.

All the experience with partition properties shows that Theorem 4.7 and Corollary 4.8 are not optimal. The number of parts in the partitions constructed above grows exponentially. It seems to us that the number of parts may be reduced considerably, but we do not know how to do it at present. Also this "tail" $\frac{(k-2)(k-1)}{2}$ does not seem to be really necessary, but we did not work out the technicalities for getting rid of this. Moreover, there is a huge gap between the upper bound from [3] and Corollary 4.8 .

Note. When this paper was being refereed, we wrote another paper [11, where we define several variations of the notion of largeness in the sense of Hardy hierarchy and prove Ratajczyk's main lemma for each of them. The problem of working out some variants of Hardy hierarchy is also considered in [4. In our opinion the notion of largeness suggested by the estimation lemma (called estimative largeness in [11]), in particular the work with pseudonorm rather than with fundamental sequences will be most convenient in this sort of combinatorics.

When Andreas Weiermann read this paper, he used ideas in it to prove Ackermannian lower bounds for diagonally iterated dense $(2,2)$ sets. His proof will appear in $[23$.

\section{ADDED IN PROOF}

After this paper was accepted we wrote the paper [12 in which we show that for the price of multiplying the number of parts by 3 we may construct partitions all of whose homogeneous sets are considerably smaller than the ones constructed above. Also, the number of parts may always be reduced to 2 , but the price is that we must increase the dimension by 1 .

\section{REFERENCES}

[1] Avigad, J., Sommer, R., A model theoretic approach to ordinal analysis, Bulletin of Symbolic Logic, 3 (1997), no. 1, 17-52. MR1444913 (98g:03126)

[2] Bigorajska, T., Kotlarski, H., A partition theorem for $\alpha$-large sets. Fund. Math. 160, 1999, pp. 27-37. MR1694401 (2000d:05012)

[3] Bigorajska, T., Kotlarski, H., Some combinatorics involving $\xi$-large sets. Fund. Math. 175, 2002, pp. 119-125. MR1969630(2005a:05209)

[4] Buchholz, W., Cichon, A., Weiermann, A., A uniform approach to fundamental sequences and hierarchies, Mathematical Logic Quarterly 40, 1994, pp. 273-286. MR.1271290(95g:03035a)

[5] Cholak, P., Jockush, C., Slaman, T., On the strength of Ramsey's theorem for pairs, Jour. Symb. Log. 66, March 2001, pp. 1-55. MR.1825173 (2002c:03094) 
[6] Cichoń, E. A., A short proof of two recently discovered independence results using recursion theoretic methods. Proc. Amer. Math. Soc., 87 (1983), no. 4, 704-706. MR0687646 (84f:03049)

[7] Fairtlough, M. V. H., Wainer, S. S., Ordinal complexity of recursive definitions. Information and computation, 99(2), 1992, pp. 123-153. MR1172210(93h:03059)

[8] Fairtlough, M. V. H., Wainer, S. S., Hierarchies of provably recursive functions, in: S. Buss (editor) Handbook of proof theory, Studies in Logic 137, North-Holland Publishing Company, 1998. MR1640327 (2000a:03063)

[9] Graham, R., Rothschild, B., Spencer, J., Ramsey theory. 2nd edition. Wiley, 1990. MR:1044995 (90m:05003)

[10] Ketonen, J., and Solovay, R., Rapidly growing Ramsey functions. Annals of Mathematics 113, 1981, pp. 267-314. MR0607894 (84c:03100)

[11] Kotlarski, H., Piekart, B., Some variations of Hardy hierarchy, Math. Log. Quart. 51, 2005, pp. 417-434. MR2150370 (2006a:03080)

[12] Kotlarski, H., Piekart, B., Weiermann, A., More on lower bounds for partitioning $\alpha$-large sets, submitted to Annals of Pure and Applied Logic.

[13] Kotlarski, H., and Ratajczyk, Z., Inductive full satisfaction classes. Ann. Pure and Appl. Log., 47, 1990, pp. 199-223. MR.1058297(92b:03058)

[14] Kotlarski, H., and Ratajczyk, Z., More on induction in the language with a satisfaction class. Zeitschr. Math. Log., 36, 1990, pp. 441-454. MR1090318 (92b:03059)

[15] Kuratowski, K., Mostowski, A., Set theory. Państwowe Wydawnictwo Naukowe, 1976.

[16] Loebl, M., Nešetřil, J., An unprovable Ramsey-type theorem, Proc. Amer. Math. Soc. 116, 1992, pp. 819-824. MR.1095225 (93a:03067)

[17] Paris, J., Harrington, L., A mathematical incompleteness in Peano arithmetic, in: Handbook of mathematical logic (J. Barwise, editor), North-Holland Publ. Comp. 1977, pp. 1133-1142.

[18] Ratajczyk, Z., A combinatorial analysis of functions provably recursive in $I \Sigma_{n}$. Fund. Math. 130, 1988, pp. 191-213. MR0970904 (90d:03126)

[19] Ratajczyk, Z., Subsystems of true arithmetic and hierarchies of functions. Ann. Pure and Appl. Log. 64, 1993, pp. 95-152. MR1241251 (94f:03068)

[20] Sommer, R., Transfinite induction and hierarchies of functions generated by transfinite recursion within Peano arithmetic, Ph.D. thesis, University of California, Berkeley, 1990.

[21] Sommer, R., Transfinite induction within Peano Arithmetic, Ann. Pure and Appl. Log. 76, 1995, pp. 231-289. MR1366513 (97f:03075)

[22] Weiermann, A., A classification of rapidly growing Ramsey functions, Proc. Amer. Math. Soc. 132, No. 2, pp. 553-561. MR2022381 (2004m:03211)

[23] Weiermann, A., Classifying the phase transition for $\operatorname{DENSE}(2,2)$, in preparation.

Faculty of Mathematics, Cardinal Stefan Wyszyński University, ul. Dewajtis 5, 01 815 WarszaWa, POLAND

E-mail address: tebigo@op.pl

Faculty of Mathematics, Cardinal Stefan Wyszyński University, ul. Dewajtis 5, 01 815 Warszawa, Poland - and - Institute of Mathematics, Polish Academy of Sciences, ul. Śniadeckich 8, P.O. Box 137, 00-950 Warszawa, Poland

E-mail address: hkl@impan.gov.pl 